\title{
Avaliação da Energética Prevista nos Modelos Regionais BRAMS, ETA e WRF: Parte I - Inverno
}

\author{
Manoel A. Gan ${ }^{1}$ (D), Sérgio H.S. Ferreira ${ }^{1}$, Everson D. Piva ${ }^{2}$, José R. Rozante ${ }^{1}$, José R.M. Garcia ${ }^{1}$, \\ Alex A. Fernandes ${ }^{1}$, André L.O. Neves ${ }^{1}$, Vinícios M. Silva ${ }^{1}$ \\ ${ }^{1}$ Centro de Previsão de Tempo e Estudos Climáticos, Instituto Nacional de Pesquisas Espaciais, \\ São José dos Campos, SP, Brasil. \\ ${ }^{2}$ Departamento de Física, Centro de Ciências Naturais e Exatas, Universidade Federal de Santa \\ Maria, Santa Maria, RS, Brasil.
}

Recebido em: 25 de Maio de 2021 - Aceito em: 3 de Agosto de 2021

\begin{abstract}
Resumo
O objetivo deste estudo é avaliar os termos da equação da tendência da energia cinética do distúrbio ( $\left.K^{\prime}\right)$ nas previsões de $24 \mathrm{~h}$ e $48 \mathrm{~h}$ dos modelos regionais BRAMS, WRF e ETA. Estes modelos foram integrados operacionalmente no Centro de Previsão de Tempo e Estudos Climáticos (CPTEC). O período de avaliação foi de 1 de junho a 31 de agosto de 2016, sendo que o domínio utilizado foi a região da América do Sul. A resolução horizontal dos modelos é de $5 \mathrm{~km}$ e a temporal é de 6 horas. As condições iniciais e de contorno foram obtidas do Global Forecast System (GFS) do National Centers for Environmental Prediction (NCEP), com resolução horizontal de $0,25^{\circ}$. Os modelos foram inicializados apenas com a análise das 1200 UTC. Os modelos foram integrados com a versão não-hidrostática, sendo que o WRF utilizou a parametrização cumulus de Kain-Frisch, o ETA a parametrização culumus de Betts-Miller e o BRAMS Grell e Freitas. Em geral, os três modelos subestimam $K$ ' nas latitudes médias, principalmente sobre os oceanos, porém o ETA é o que menos subestima e o WRF o que tem o maior viés negativo. Com relação aos termos de conversão baroclínica e barotrópica, além do termo de convergência do fluxo ageostrófico, os três modelos apresentam um padrão semelhante aos das análises numéricas do GFS.
\end{abstract}

Palavras-chave: energia cinética, avaliação, modelos regionais.

\section{Evaluation of the Forecasts of the Energetics in the BRAMS, ETA and WRF Regional Models: Part I - Winter}

\begin{abstract}
The aim of this study is to evaluate the terms of the eddy kinetic energy $\left(K^{\prime}\right)$ equation in the $24 \mathrm{~h}$ and $48 \mathrm{~h}$ forecasts of the regional models BRAMS, WRF and ETA. These models were operationally integrated at the Weather Forecast and Climate Studies Center (CPTEC). The evaluation period was from June 1 to August 31, 2016 and the domain was the region of South America. The horizontal resolution of the three models is $5 \mathrm{~km}$ and the temporal is $6 \mathrm{~h}$. The initial and boundary conditions were obtained from the Global Forecast System (GFS) of the National Centers for Environmental Prediction (NCEP), with a horizontal resolution of $0.25^{\circ}$. The models were initialized only with the analysis of 1200 UTC. The non-hydrostatic version of the three models was run, with WRF using Kain-Frisch cumulus parameterization, ETA Betts-Miller culumus parameterization and BRAMS Grell and Freitas. In general, the three models underestimate $K^{6}$ in mid-latitudes, mainly over the oceans, however, ETA is the one that underestimates the least and WRF the most. Regarding the terms of baroclinic and barotropic conversion, in addition to the term of ageostrophic flow convergence, the three models have a similar pattern of these three terms with that of GFS numerical analysis.
\end{abstract}

Keywords: kinetic energy, evaluation, regional models.

Autor de correspondência: Manoel Alonso Gan, manoel.gan@inpe.br. 


\section{Introdução}

Ao longo do ano a atmosfera na América do Sul é influenciada por sistemas transientes, que alteram a circulação atmosférica e determinam as condições do tempo sobre o continente e oceanos adjacentes. Dentre os sistemas transientes têm-se os sistemas frontais, associados a um anticiclone frio, que se deslocam do oceano Pacífico Sul, passam pela Argentina e seguem para o nordeste do Brasil (Kousky, 1979; Oliveira, 1986; Rodrigues et al., 2004; Andrade, 2005); ciclones extratropicais associados aos sistemas frontais e os que se desenvolvem na região devido a condições ciclogenéticas originadas na própria região (Gan e Rao, 1991; Reboita, 2008; Reboita et al., 2009), ciclones subtropicias (Reboita, 2008; Reboita et al., 2009), e vórtices ciclônicos ou cavados em altos níveis sobre a costa oeste da América do Sul (Funatsu et al., 2004, Iwabe e Da Rocha, 2009, Fuenzalida et al., 2005; Campetella and Possia, 2007, Pinheiro, 2010) e sobre o Nordeste do Brasil (Kousky e Gan 1981; Moraes, 2016); Sistemas Convectivos de Mesoescala (SCMs - Figueiredo e Scolar, 1996, Anabor et al., 2009) e sistemas ciclônicos em níveis médios conhecidos como vírgula invertida (Bonatti e Rao, 1987; Hallak, 2000). Quando esses sistemas atuam na região, alterações no tempo podem ocorrer como queda acentuada de temperatura, precipitação intensa e ventos severos.

As principais teorias de geração e intensificação de sistemas transientes nas latitudes médias são a da instabilidade baroclínica, instabilidade barotrópica e o Desenvolvimento Corrente Abaixo (DCA), além da Instabilidade Não-modal e o Desenvolvimento Transiente (NielsenGammon, 1995). Porém neste estudo não serão analisadas essas duas últimas. A instabilidade baroclínica é baseada nas trocas de energia que ocorrem entre o distúrbio e o escoamento médio, através do fluxo horizontal e vertical de calor (Lorenz, 1967). Já a instabilidade barotrópica está associada a processos não lineares, tal como transporte horizontal de momentum, associados ao cisalhamento horizontal do vento (Lorenz, 1967). No caso do DCA, um sistema pode crescer recebendo energia de um sistema localizado corrente acima (a oeste), através de fluxos ageostróficos (Orlanski e Katzfey, 1991).

Uma teoria que associa o DCA à instabilidade baroclínica é o Desenvolvimento Corrente Abaixo Baroclínico (DCAB), proposta por Orlanski e Sheldon (1993). Nessa teoria, o crescimento inicial da energia cinética do distúrbio ocorre por convergência de fluxos ageostróficos (DCA), mas em uma segunda etapa é mantido por instabilidade baroclínica. A ideia dessa teoria, é que o distúrbio gerado por fluxos ageostróficos em altos níveis induz uma circulação em baixos níveis. Esta indução aumenta o gradiente de temperatura ao redor do sistema, fazendo com que o distúrbio utilize a baroclinia existente localmente para continuar intensificando (Orlanski e Sheldon, 1993).
Um dos métodos utilizados para realização de tais estudos é a análise de geração e conversões de energia, ou análise energética; tais como nos trabalhos de Orlanski e Katzfey (1991), Gan e Rao (1999), Chang (2000), Piva et al. (2010), Ferreira (2013), Gan e Piva (2013, 2016). Existem várias formulações empregadas para determinação de energética, sendo uma das mais utilizadas é a proposta no estudo de Orlanski e Katzfey (1991).

Em comparação aos métodos convencionais de análise sinótica, o uso de análise energética, possui a vantagem de permitir avaliar diretamente as principais conversões de energia envolvidas, tais como conversão baroclínica, barotrópica e convergência do fluxo ageostrófico. Outra vantagem é incorporar aos resultados as componentes da energética integradas verticalmente; dando assim, uma melhor visão dos sistemas meteorológicos, sem que haja a necessidade de analisar individualmente diversas camadas da atmosfera. Os resultados podem ainda ser integrados no volume em torno do sistema meteorológico de interesse, a fim de acompanhar as várias etapas da evolução das componentes de energia que favorecem a intensificação e a dissipação dos sistemas.

Devido a energética ser uma componente importante na circulação geral da atmosfera, vários estudos foram realizados para entender as conversões de energia cinética $(K)$ do estado básico para a perturbação e vice-versa. Alguns estudos foram realizados para delinear a dinâmica da ciclogênese de superfície (Piva et al., 2010; Rosa et al., 2013) e dos vórtices ciclônicos em altos níveis (Gan e Piva, 2013, 2016). Orlanski e Katzfey (1991), estudando o ciclo de vida dos ciclones do Hemisfério Sul, destacaram a importância da convergência do fluxo ageostrófico na evolução do ciclone. Chang (2000) mostrou a importância do DCA para a formação de cavados corrente abaixo no escoamento em nível superior no verão do hemisfério sul.

Embora os modelos de previsão de tempo tenham uma boa destreza em prever o campo de vento e, portanto a $K$ dos Distúrbios ( $\left.K^{\prime}\right)$, é importante avaliar se os modelos também têm um bom desempenho em prever os termos da equação da tendência da $K^{\prime}$. Assim, o objetivo deste estudo é avaliar a destreza dos modelos BRAMS, ETA e WRF em prever com antecedência de 24 e 48 horas a $K^{\prime}$ e os termos de conversão baroclínica e barotrópica, além do termo de convergência do fluxo ageostrófico. O período selecionado para o estudo foi de $1^{\circ}$ de junho a 31 de agosto de 2016, para a região da América do Sul. Esse período foi escolhido, pois em 2017 o CPTEC integrou esses modelos com a resolução espacial de $5 \mathrm{~km}$ e com a configuração que eles eram rodados diariamente de forma operacional. $\mathrm{O}$ período integrado para a avaliação foi de 1 de março de 2016 a 28 de fevereiro de 2017, mas para o presente estudo foram escolhidos os três meses de inverno por ser a estação do ano em que os ventos em altos níveis são mais intensos. 


\section{Dados e Metodologia}

A metodologia empregada para avaliar os termos de equação da tendência de $K^{\prime}$ na previsão dos modelos BRAMS, ETA e WRF, será apresentada neste item. O domínio avaliado é a região da América do Sul. Vale ressaltar que neste estudo serão avaliadas somente as previsões de $24 \mathrm{~h}$ e $48 \mathrm{~h}$.

A configuração dos modelos é aquela que era utilizada para a previsão de tempo operacional do CPTEC. Rodou-se a versão não-hidrostática dos três modelos, sendo que o BRAMS utilizou Grell e Freitas (2014) para a parametrização cumulus, o ETA usou Betts-Miller e o WRF usou Kain-Frisch. As condições iniciais e de contorno foram obtidas do Global Forecast System (GFS) do National Centers for Environmental Prediction (NCEP), com resolução horizontal de $0,25^{\circ}$, sendo que a de contorno lateral foi inserida a cada $6 \mathrm{~h}$. Os modelos foram inicializados apenas com a análise das 1200 UTC (mais informações sobre a configuração dos modelos podem ser vistas na Tabela 1$)$.

Para determinar de forma objetiva a destreza dos modelos avaliados, calculou-se o viés e a raiz do erro médio quadrático (root mean square error, RMSE (sigla em inglês), daqui em diante).

A formulação empregada para calcular os termos da equação da tendência de $K$ ' foi aquela desenvolvida por Orlanski e Katzfey (1991) e modificada por Chang (2000) (Eq. (1)). Esta formulação tem sido usada para vários estudos como os de Gan e Piva $(2013,2016)$ para vórtices ciclônicos em altos níveis sobre o oceano Pacífico Sul e Müller et al. (2017) para eventos de geadas ocorridas na Argentina, pois apresenta as principais teorias de geração e intensificação dos sistemas transientes, a saber: Instabilidade Baroclínica (BRC, termo 3), Desenvolvimento Corrente Abaixo (DCA, termo 2) e Instabilidade Barotrópica (BRT, termo 4).

$$
\begin{gathered}
\frac{\partial\left\langle K^{\prime}\right\rangle}{d t}=-\underbrace{\left\langle\nabla \cdot \boldsymbol{V} K^{\prime}\right\rangle}_{1}-\underbrace{\left\langle\nabla \cdot \boldsymbol{V}_{\boldsymbol{a}} \boldsymbol{\phi}^{\prime}\right\rangle}_{2}-\underbrace{\left\langle\omega^{\prime} \alpha^{\prime}\right\rangle}_{3}-\underbrace{\left\langle\boldsymbol{V}^{\prime} \cdot\left(\boldsymbol{V}_{3}^{\prime} \cdot \nabla_{3}\right) \overline{\boldsymbol{V}}+\boldsymbol{V}^{\prime} \cdot \overline{\left(\boldsymbol{V}_{3}^{\prime} \cdot \nabla_{3}\right) \boldsymbol{V}^{\prime}}\right\rangle}_{4}+\underbrace{\left\langle\nabla \cdot \boldsymbol{V}_{\boldsymbol{v}} K^{\prime}\right\rangle}_{5}+\underbrace{\left[\left(\frac{d P_{s}}{d t}+\boldsymbol{V}_{\boldsymbol{v}} \cdot \nabla \mathrm{P}_{s}\right) K^{\prime}\right]_{b}}_{6} \\
-\underbrace{\left[\omega K^{\prime}\right]_{b}}_{7}+\underbrace{\left[\omega K^{\prime}\right]_{t}}_{8}-\underbrace{\left[\omega^{\prime} \phi^{\prime}\right]_{b}}_{9}+\underbrace{\left[\omega^{\prime} \phi^{\prime}\right]_{t}}_{10}+\left\langle R^{\prime}\right\rangle
\end{gathered}
$$

onde $K$ ' é a energia cinético do distúrbio, $\phi$ o geopotencial, $\mathbf{V}$ o vento horizontal e $\omega$ o movimento vertical em coordenadas de pressão. Os limites para integração vertical (indicada pelos símbolos $<>$ ) são definidos em coordenadas de pressão, indo do nível de pressão da base da coluna vertical $\left(P_{\mathrm{s}}\right)$ ao nível de pressão do topo $\left(P_{\mathrm{t}}\right)$, onde $P_{\mathrm{s}}$ corresponde ao nível dos dados mais próximo da superfície, mas que não intercepta a superfície da Terra no ponto considerado. O nível $P_{\mathrm{t}}$ é fixado em $100 \mathrm{hPa}$ de forma a conter toda a troposfera. O símbolo [ ] representa a integral de superfície ( $\mathrm{t}$ - topo e b - base), o subscrito "a" indica a componente ageostrófica, o subscrito " $\mathrm{v}$ " indica a velocidade de deslocamento do volume e 3 é o número de dimensões. A barra significa a média temporal de 30 dias, ' a perturbação e R' o resíduo.

\section{Resultados}

O campo espacial de $K^{\prime}$ integrada verticalmente para o inverno (junho, julho e agosto) de 2016 da análise numérica do GFS, interpolada para uma grade de $5 \mathrm{~km}$ de latitude e longitude, mostra valores mais intensos de $K^{\prime}$ ao sul de $20^{\circ} \mathrm{S}$, com um centro de máximo valor sobre o oceano Atlântico Sul em torno de $45^{\circ} \mathrm{S}-30^{\circ} \mathrm{W}$ (Fig. 1a), um outro máximo se localiza ao longo dos Andes e um terceiro sobre o oceano Pacífico Sul, que se estende para a região central da Argentina. Apesar da retirada da montanha no cálculo da integração vertical, o efeito topográfico se faz presente nesse campo com o máximo ao longo dos Andes. Ao analisar subjetivamente o mesmo campo da análise e das previsões dos três modelos para $24 \mathrm{~h}$ (Figs. 1b, 1c e 1d) e $48 \mathrm{~h}$ (Figs. 2b, 2c e 2d), pode-se notar que, em geral, os três modelos apresentam um padrão semelhante, sendo o ETA o modelo que mais se aproxima da análise, possuindo valores simulados ligeiramente inferiores, porém maiores do que os das previsões dos outros dois modelos (BRAMS e WRF), principalmente no setor oeste do oceano Atlântico.

O campo da análise GFS do temo BRC mostra valores positivos na região ao sul de $20^{\circ} \mathrm{S}$ e a leste de $65^{\circ} \mathrm{W}$ (Fig. 3a). Parece haver uma banda de valores positivos do termo BRC que se inicia sobre o Uruguai e se estende para sudeste em direção ao oceano Atlântico. Esses valores positivos estão localizados sobre o Uruguai, região esta de máximo de ciclogênese extratropical identificada por Gan e Rao (1991). Essa região de valores positivos se estende sobre parte da região do Atlântico Sul, para onde os ciclones formados sobre o Uruguai propagam (Reboita, 2008; Reboita et al., 2009). As previsões de $24 \mathrm{~h}$ dos três modelos apresentam um padrão muito semelhante ao da análise (Figs. 3b, 3c e 3d), porém os três modelos (Figs. 3b, 3c e 3d) mostram, em alguns setores da região 
Tabela 1 - Configurações dos modelos BRAMS, ETA e WRF.

\begin{tabular}{|c|c|c|c|}
\hline & BRAMS & ETA & WRF \\
\hline $\begin{array}{l}\text { Parametrização cúmu- } \\
\text { los profundos }\end{array}$ & Grell e Freitas (2014) & $\begin{array}{l}\text { Betts-Miller (Betts and Miller 1986; Janjic } \\
\text { 1994) }\end{array}$ & $\begin{array}{l}\text { Modified Kain-Fritsch with } \\
\text { trigger based on PDF }\end{array}$ \\
\hline $\begin{array}{l}\text { Parametrização cúmu- } \\
\text { los rasos }\end{array}$ & Grell e Dévényi (2002) & Faz parte do esquema de Betts-Miller & $\begin{array}{l}\text { Faz parte do esquema de } \\
\text { Kain-Frisch }\end{array}$ \\
\hline Microfísica (MP) & 2-moment (original BRAMS) & Ferrier (Ferrier et al., 2002) & $\begin{array}{l}\text { WSM 6-class graupel } \\
\text { Scheme (WSM6) }\end{array}$ \\
\hline Processos de superfície & JULES (Moreira et al., 2013) & NOAH (Ek et al., 2003) & Unified NOAH \\
\hline $\begin{array}{l}\text { Radiação de Onda Curta } \\
\text { (ROC) }\end{array}$ & RRTMG (Mlawer et al., 1997; Iacono et al., 2008) & Lacis and Hansen (Lacis and Hansen 1974) & RRTMG Scheme \\
\hline $\begin{array}{l}\text { Radiação de Onda } \\
\text { Longa (ROL) }\end{array}$ & RRTMG (Mlawer et al., 1997; Iacono et al., 2008) & $\begin{array}{l}\text { Fels and Schwarzkopf (Fels and Schwarz- } \\
\text { kopf 1975) }\end{array}$ & RRTMG Scheme \\
\hline $\begin{array}{l}\text { Turbulência e Camada } \\
\text { Limite }\end{array}$ & Mellor and Yamada (Mellor and Yamada, 1982) & $\begin{array}{l}\text { Mellor-Yamada } 2.5 \text { e Mellor-Yamada } 2.0 \\
\text { (Mellor and Yamada 1982; Janjic 1990) }\end{array}$ & YSU Scheme \\
\hline Pontos na direção $\mathrm{x}$ & 1360 & 913 & 830 \\
\hline Pontos da direção y & 1480 & 2113 & 889 \\
\hline Coordenadas na vertical & Sigma-z & Eta (Mesinger 1984) & $\begin{array}{l}\text { Mass-based terrain } \\
\text { following coordinate }\end{array}$ \\
\hline $\begin{array}{l}\text { Número de níveis na } \\
\text { vertical }\end{array}$ & 45 & 50 & 42 \\
\hline $\begin{array}{l}\text { Número de níveis no } \\
\text { solo }\end{array}$ & 7 & 4 & 4 \\
\hline grade horizontal & Arakawa C-grid & E de Arakawa & Arakawa C-grid \\
\hline esquema de advecção & $\begin{array}{l}\text { Forward upstream of second order (Tremback } \\
\text { et al., 1987) }\end{array}$ & Finite Volume (Mesinger et al., 2012) & $\begin{array}{c}\text { Positivo-definido de } 2 \mathrm{a} \text { a } 6 \mathrm{a} \\
\text { ordem }\end{array}$ \\
\hline Passo de tempo & $12 \mathrm{~s}$ & $10 \mathrm{~s}$ & $\begin{array}{l}\text { Adaptativo (mínimo } 18 \mathrm{~s} \mathrm{e} \\
\text { máximo } 54 \mathrm{~s} \text { ) }\end{array}$ \\
\hline Esquema de topografia & Average orography & Steps with cutcell (Mesinger et al., 2012) & 4-point interpolation \\
\hline Mapa de topografia & USGS (1 km de resolução) (Gesch et al., 1999) & USGS $90 \mathrm{~m}$ & GMTED 2010 \\
\hline Mapa de uso do solo & $\begin{array}{l}\text { OGE (fora do Brasil) + IBGE/INPE (no Brasil) } \\
\text { (1 km de resolução) (Olson, 1994; Sestini et al., } \\
\text { 2003) }\end{array}$ & Doyle (Doyle et al., 2013) & 24-category USGS landuse \\
\hline Tipo de solo & FAO-INPE (55 km de resolução) (Zobler, 1999) & 18 tipos & 16-category BNU \\
\hline Umidade do solo & $\begin{array}{c}\text { CPTEC/INPE (28 km de resolução) (Gevaerd and } \\
\text { Freitas, 2006) }\end{array}$ & Climatológica & GFS \\
\hline SST & $\begin{array}{l}\text { Semanal do NCEP (111 km de resolução) (Rey- } \\
\text { nolds et al., 2002) }\end{array}$ & Semanal do NCEP & Semanal do NCEP \\
\hline Equações básicas & Não-hidrostático & Não-hidrostático & Não-hidrostático \\
\hline
\end{tabular}

tropical da América do Sul, valores positivos, sendo os valores do WRF ligeiramente maiores. É observado também, tanto na análise quanto nas previsões, um padrão de valores negativos a oeste dos Andes e valores positivos a leste. Provavelmente este padrão se deve ao levantamento orográfico de ar frio a oeste dos Andes e subsidência no lado leste. Estudos mostraram que a oeste dos continentes, a atuação de anticiclones subtropicais estabelece uma atmosfera com temperatura relativamente mais baixa (Seager et al., 2004; Liu et al., 2004). Esse ar frio, forçado a cruzar os Andes, gera um termo baroclínico negativo, pois tem ascensão de ar frio, mas ao descer no lado sotavento dos Andes torna-se positivo. Esse comportamento é mais forte nos modelos BRAMS e WRF, indicando que devem existir problemas no cálculo do movimento vertical próximo às montanhas. Resultado semelhante é observado para as previsões de $48 \mathrm{~h}$ (Figs. 4b, 4c e 4d), porém os valores positivos sobre o noroeste da América do Sul previsto pelos modelos BRAMS e WRF são maiores (Figs. 4b e $4 \mathrm{~d}$, respectivamente) do que nas previsões de $24 \mathrm{~h}$ (Figs. 3b e $3 \mathrm{~d}$, respectivamente).

$\mathrm{O}$ termo BRT (Figs. 5 e 6 ) mostra valores bem inferiores aos da conversão baroclínica (Figs. 3 e 4), porém observa-se na análise e nas previsões de $24 \mathrm{~h}$ e $48 \mathrm{~h}$ dos três modelos, um padrão bem semelhante, com uma região de conversão negativa a leste da costa da região sudeste do Brasil e outras duas regiões com valores positivos, sendo uma sobre o sul da Argentina e a outra na costa do 

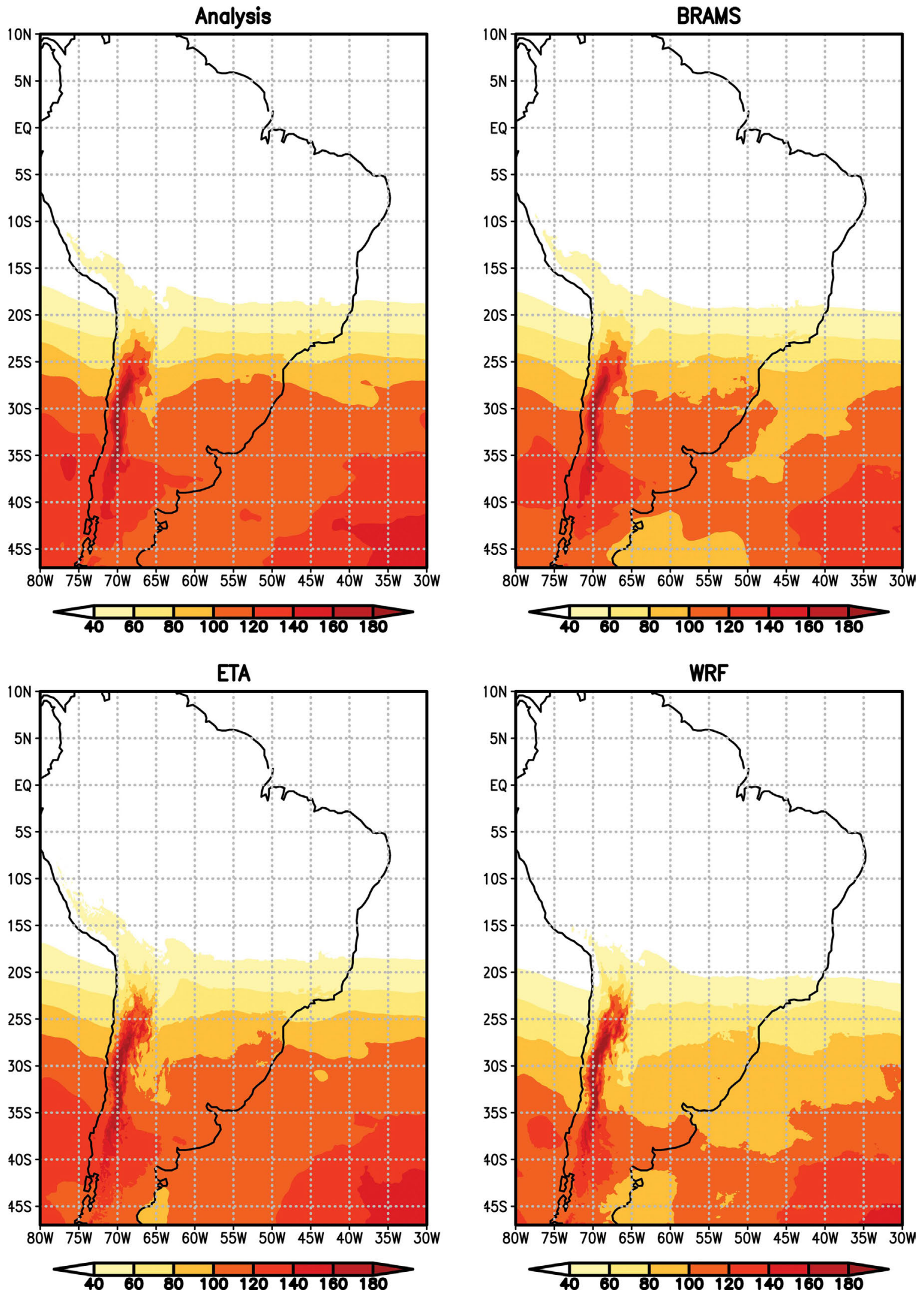

Figura 1 - Energia cinética (10 ${ }^{10}$ Joule) integrada verticalmente por unidade de massa para (a) análise e previsão de 24 h dos modelos (b) BRAMS, (c) ETA e (d) WRF para os meses de junho, julho e agosto de 2016. 

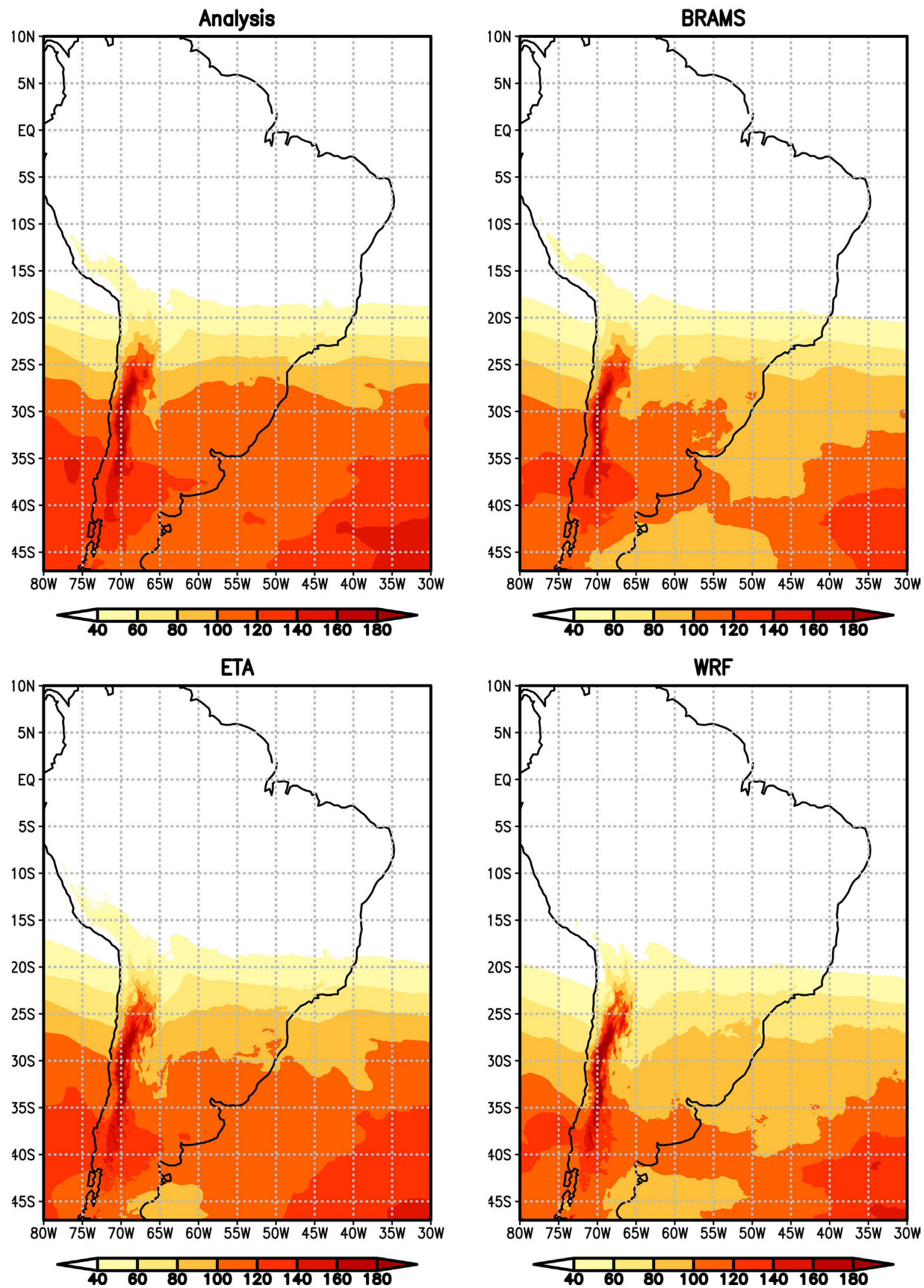

Figura 2 - Idem à Fig. 1, porém para as previsões de 48 h. 

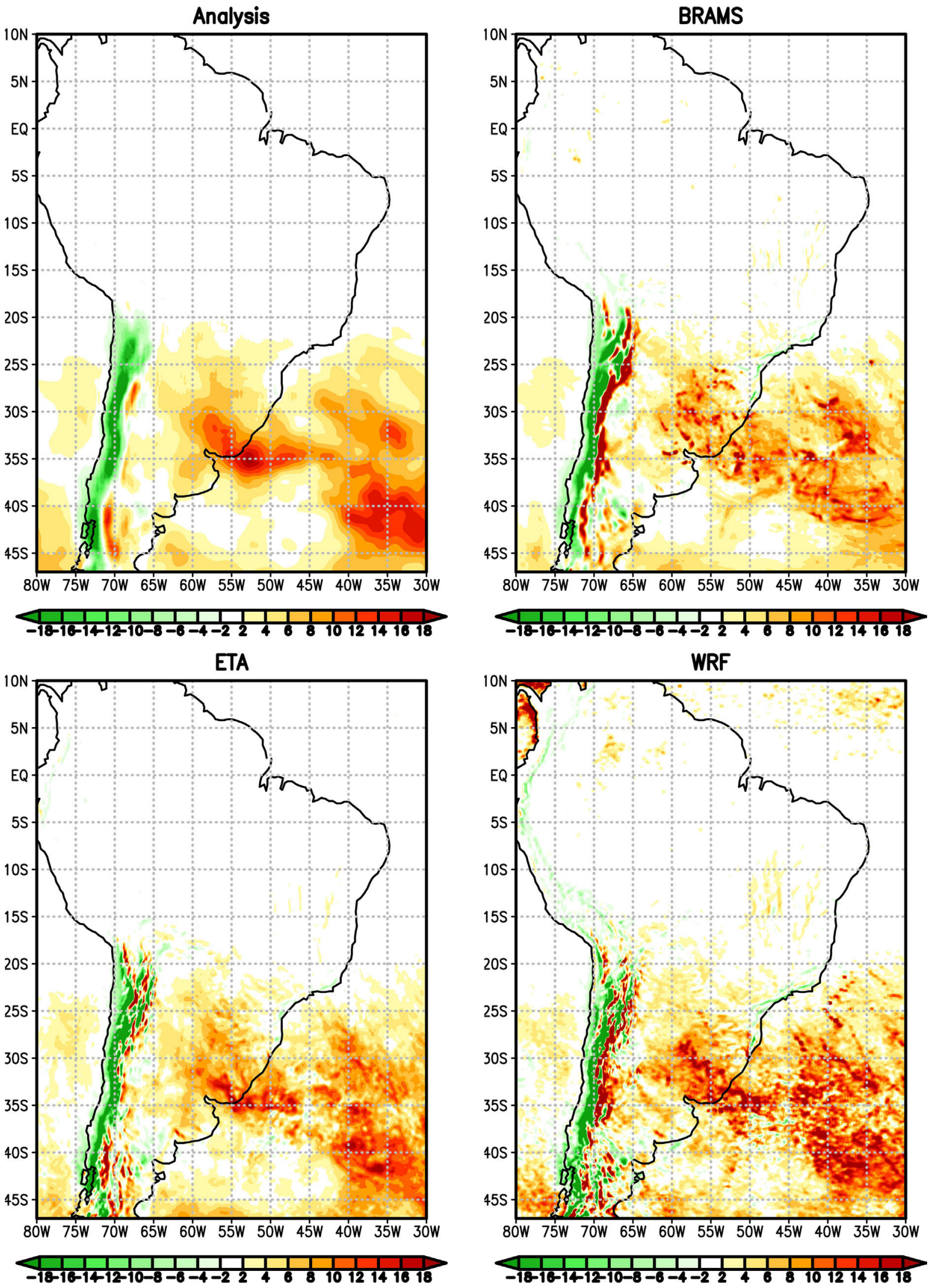

Figura 3 - Idem à Fig. 1, porém para o termo de conversão baroclínica $\left(10^{14} \mathrm{Joule} \mathrm{s}^{-1}\right)$. 

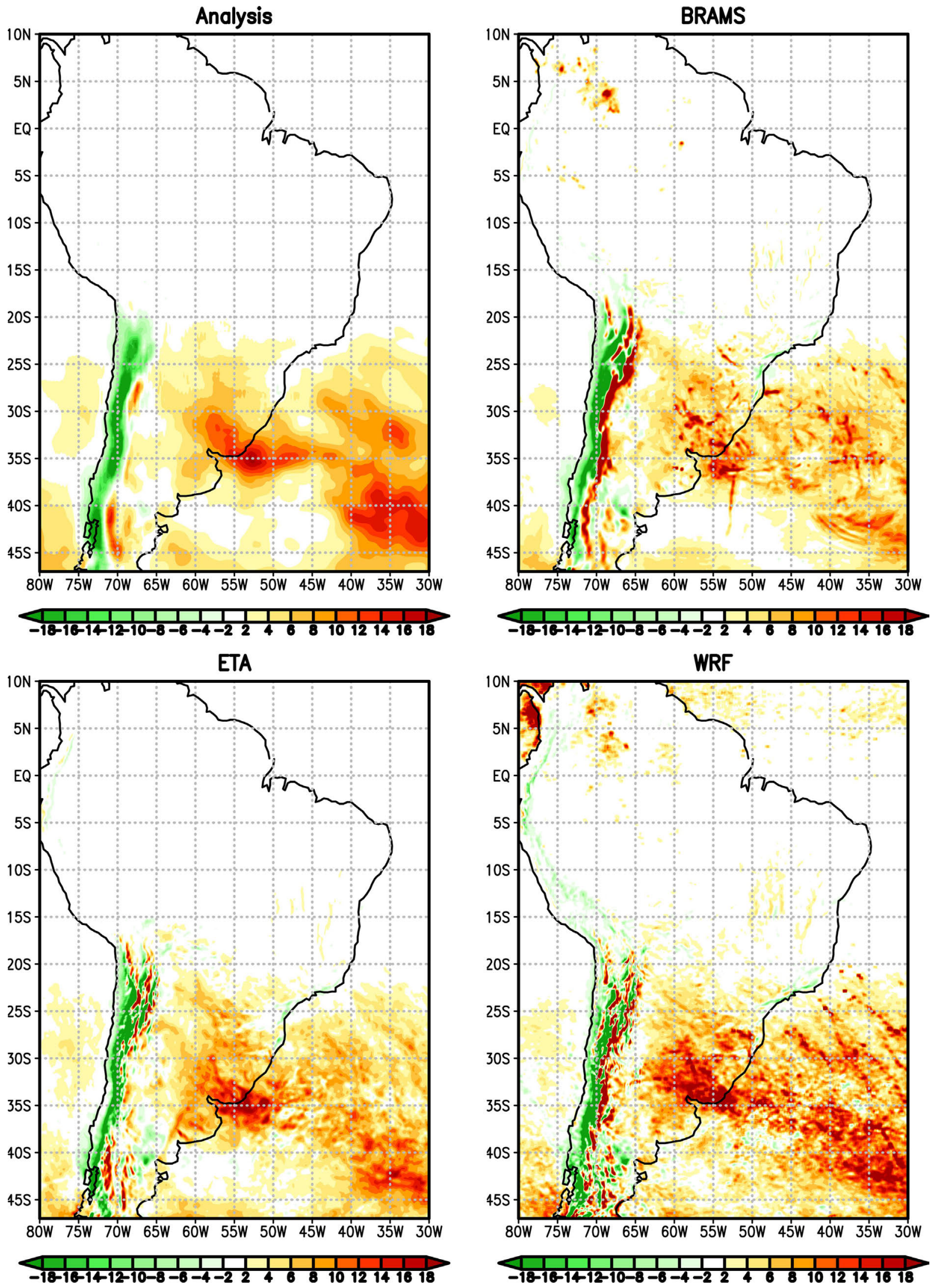

Figura 4 - Idem à Fig. 3, porém para as previsões de $48 \mathrm{~h}$. 

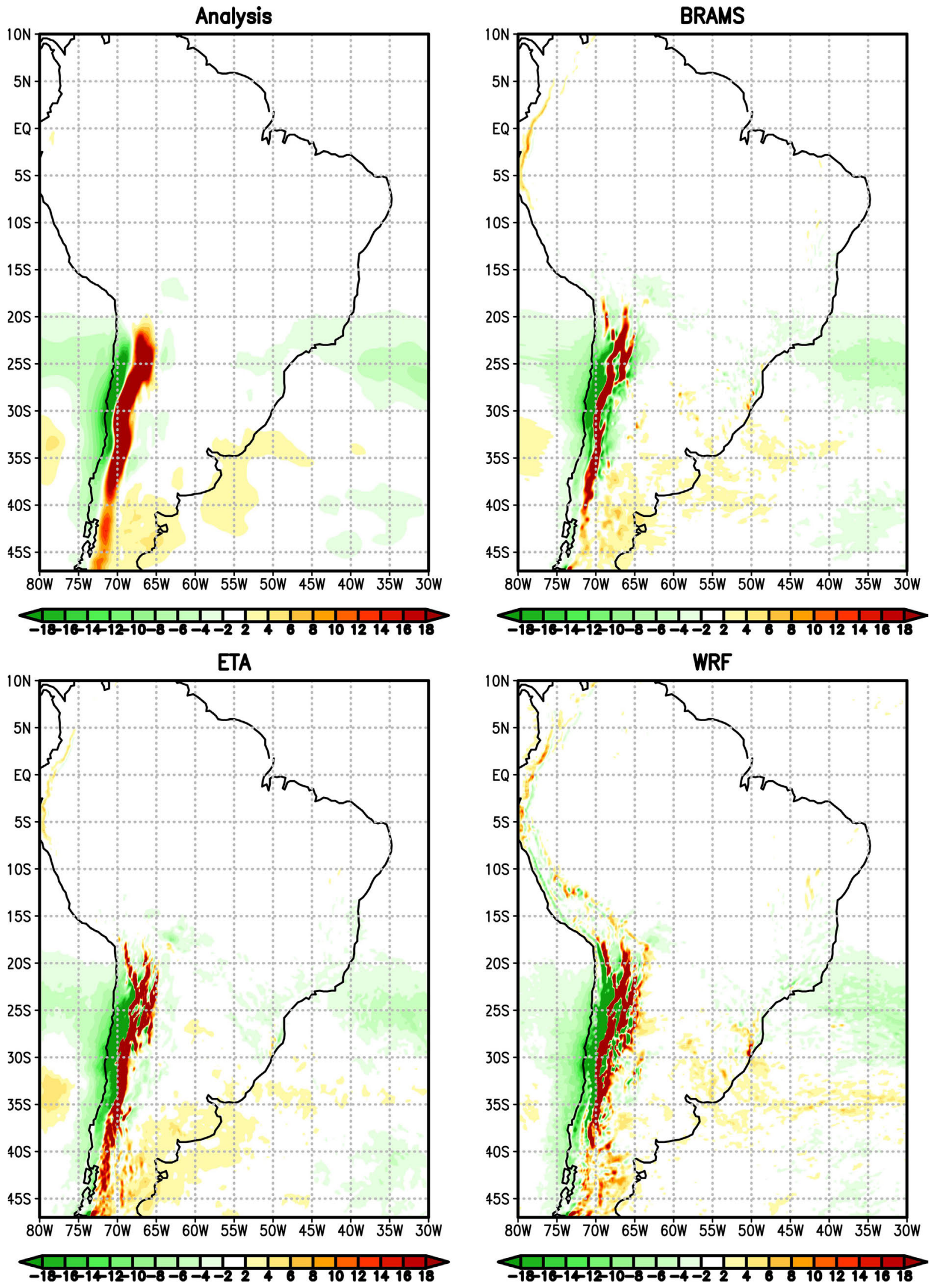

Figura 5 - Idem à Fig. 3, porém para o termo de conversão barotropica $\left(10^{14} \mathrm{Joule} \mathrm{s}^{-1}\right)$. 

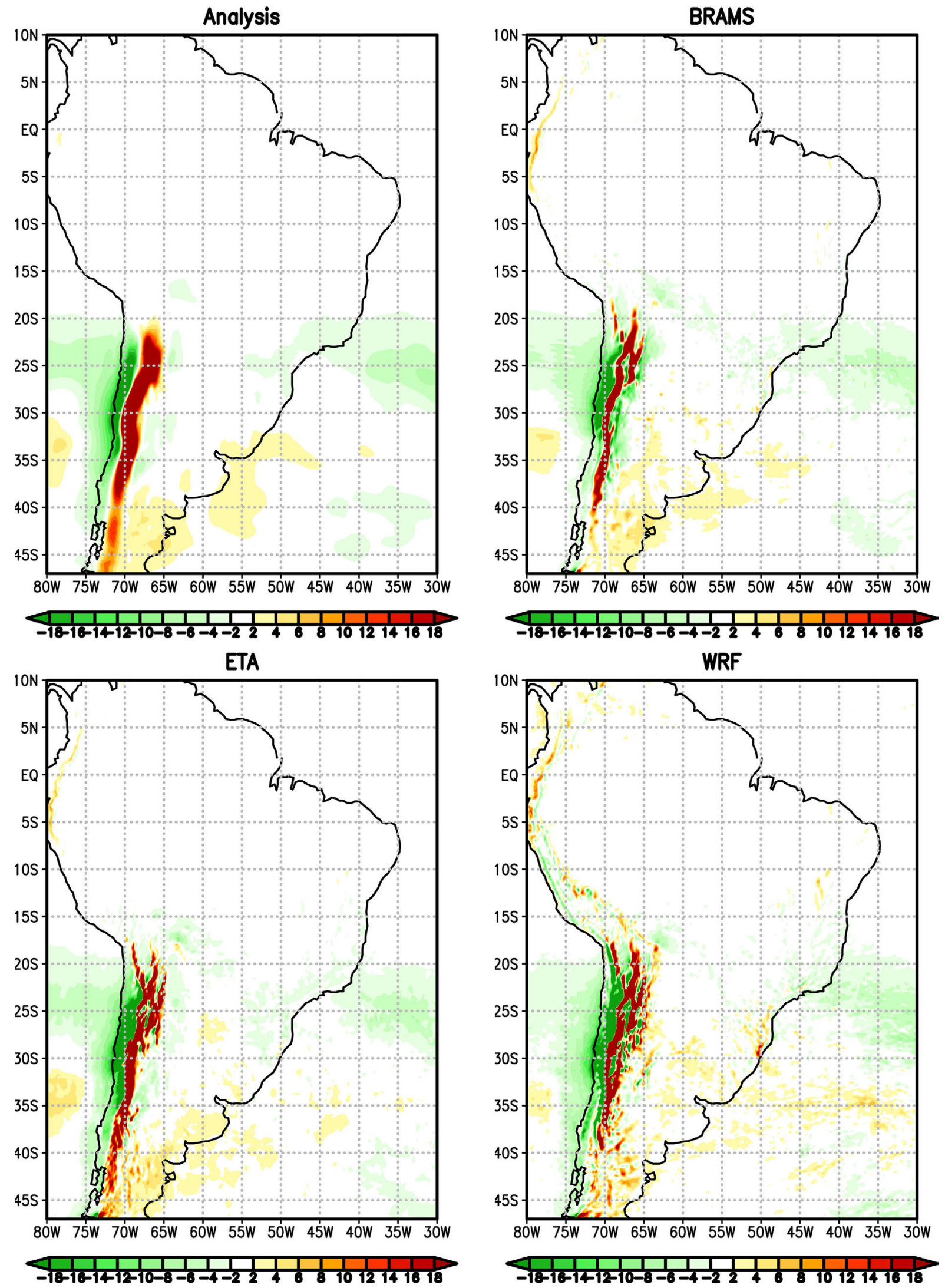

Figura 6 - Idem à Fig. 5, porém para as previsões de 48 h. 
Uruguai e da região de Buenos Aires (Argentina). A análise física desse termo não é tão simples quanto o termo baroclínico, mas pode-se pensar na sua contribuição fornecida pelo termo 4 da Eq. (1), ou seja, resumidamente sabe-se que esse termo representa a correlação entre o fluxo meridional de momentum pelo distúrbio ( $\left.u^{\prime} v^{\prime}\right)$ e o cisalhamento meridional do escoamento básico $(\mathrm{d} u / \mathrm{dy})$. Portanto, se a correlação resultar negativa (positiva) o termo BRT será positivo (negativo) e $K^{\prime}$ aumenta (reduz). Logo, o termo BRT pode ser explicado considerando a inclinação meridional das ondas em nível superior e sua posição relativa com relação ao jato subtropical. Como na região polar do jato subtropical há uma grande frequência de transientes em formação ou em desenvolvimento, os quais são caracterizados por inclinação NW-SE do eixo dos cavados em nível superior, espera-se que o fluxo meridional de momentum pelos distúrbios seja negativo, pois pode se ter $u^{\prime}<0$ e $v^{\prime}>0$, portanto $u^{\prime} v^{\prime}<0$. Por outro lado, nessa região o cisalhamento horizontal do vento deve ser positivo $(\mathrm{d} u / \mathrm{dy}>0)$. No lado equatorial do jato, isto é, sobre o sudeste do Brasil e oceano adjacente, o cisalhamento deve ser negativo $(\mathrm{d} u / \mathrm{dy}<0)$. Combinando os sinais, faz sentido, que sobre a Argentina e adjacências, o termo barotrópico seja positivo (indicando que $K^{\prime}$ aumenta), enquanto sobre o sudeste do Brasil e adjacências seja negativo (indicando que $K^{\prime}$ reduza). Ao longo dos Andes, o termo BRT parece ter influência da componente vertical do escoamento, uma vez que há valores negativos a oeste dos Andes e valores positivos a leste. Apesar dos modelos apresentarem um padrão similar, algumas pequenas diferenças são observadas, principalmente na região sul da América do Sul, onde por exemplo, as previsões de $24 \mathrm{~h}$ e de $48 \mathrm{~h}$ dos modelos ETA e o BRAMS, unem as duas regiões de conversão positiva, observadas no campo da análise.

O termo de convergência do fluxo ageostrófico mostra um padrão com valores positivos e negativos na região ao sul de $15^{\circ} \mathrm{S}$, tanto no campo da análise (Fig. 7a), como nas previsões de $24 \mathrm{~h}$ (Figs. 7b, 7c e 7d) e de $48 \mathrm{~h}$ (Figs. 8b, 8c e 8d). Assim como mostrado para os outros termos, o padrão das previsões dos três modelos se assemelha ao da análise numérica. Nota-se, no campo da análise numérica, valores negativos (divergência do fluxo ageostrótico) sobre o continente ao sul de $30^{\circ} \mathrm{S}$ e ao longo do lado leste da cordilheira dos Andes, ao sul de $20^{\circ} \mathrm{S}$. Valores positivos são observados a barlavento dos Andes ao sul de $20^{\circ} \mathrm{S}$, sobre o oceano Pacífico Sul ao sul de $35^{\circ} \mathrm{S}$, sobre o continente na faixa entre $15^{\circ}-25^{\circ} \mathrm{S}$, e oceano Atlântico a sul de $20^{\circ} \mathrm{S}$ e a leste de $45^{\circ} \mathrm{W}$. O padrão de valores positivos e negativos ao longo dos Andes sugere uma influência topográfica no escoamento ageostrófico. Importante ressaltar também que, tanto a análise, quanto às previsões, indicam que as regiões ciclogenéticas do Uruguai e Golfo de São Matias, exportam energia para várias direções, mas principalmente para nordeste e sudeste, onde há áreas de valores positivos de DCA.

Em uma análise subjetiva, nota-se claramente que as previsões do modelo WRF apresentam uma região de valores negativos sobre o oceano Atlântico Sul a leste de $40^{\circ} \mathrm{W}$ entre $30-40^{\circ} \mathrm{S}$, o que não se observa no campo da análise. Como valores negativos do termo de convergência do fluxo ageostrófico significa que há exportação de energia cinética, isso pode indicar que as previsões do modelo WRF simulam ventos mais fracos sobre essa região.

Para obter uma análise quantitativa calculou-se o viés e o RMSE para $K^{\prime}$ e os termos BRC, BRT e DCA. Analisando o campo do viés de $K^{\prime}$ dos três modelos para os dois horários, nota-se que em geral, os três modelos subestimam $K^{\prime}$ (Figs. 9, 10 e 11 - linha superior), porém o ETA é o que menos subestima e o WRF o que mais, inclusive nos trópicos. Como esperado, as previsões de $48 \mathrm{~h}$ subestimam mais do que as previsões de $24 \mathrm{~h}$.

Uma análise mais detalhada do viés dos modelos mostra que o modelo BRAMS subestimas mais $K$ ' sobre os oceanos ao sul de $15^{\circ} \mathrm{S}$, sendo que em algumas regiões da Argentina ele ligeiramente superestima, tanto nas previsões de $24 \mathrm{~h}$, quanto nas de $48 \mathrm{~h}$. Por outro lado, o modelo ETA apresenta nas previsões de $24 \mathrm{~h}$, regiões com viés positivo e negativo sobre o oceano Atlântico ao sul de $20^{\circ} \mathrm{S}$. Já sobre o continente, o modelo subestima $K^{\prime} \mathrm{em}$ algumas regiões ao sul de $20^{\circ} \mathrm{S}$, exceto ao longo dos Andes, onde aparecem regiões com viés positivo. Já o modelo WRF subestima $K^{\prime}$ em praticamente toda a região estudada, exceto ao longo dos Andes, onde aparecem regiões com viés positivo. Isso significa que quando os modelos subestimam $K^{\prime}$ na coluna atmosférica, a intensidade do vento também está sendo subestimada, sendo o modelo WRF o que mais subestima. Boer e Lambert (2008) também notaram que a energia cinética da perturbação em modelos foi mais baixa do que na análise (ou seja, reanálise do ECMWF e NCEP). Nesse estudo analisou-se o ciclo de energia de Lorenz em 12 modelos globais que participaram do AMIP2 (segunda fase do projeto de intercomparação de modelos atmosféricos). Os autores justificaram que valores menores de $K^{\prime}$ simulados pelos modelos perante as reanálises foi devido a maior dissipação de $K^{\prime}$ pelos modelos. É possível que a maior dissipação de $K^{\prime}$ nos modelos regionais possa ser a explicação para a previsão de valores menores de $K^{\prime}$ nos modelos quando comparado à análise. Essa maior dissipação de energia cinética foi identificada no WRF no estudo de Skamarock (2004). Outra explicação pode estar associada à cascata de energia, na qual os modelos poderiam transferir mais energia para as escalas menores, devido a melhor difusão e portanto as escalas maiores, como a sinótica, teriam menos $K^{\prime}$. Essa explicação é plausível, pois a resolução da análise original é de aproximadamente $25 \mathrm{~km}$, e a dos modelos é de $5 \mathrm{~km}$. Com relação aos campos de RMSE, nota-se que os três modelos apresentam 

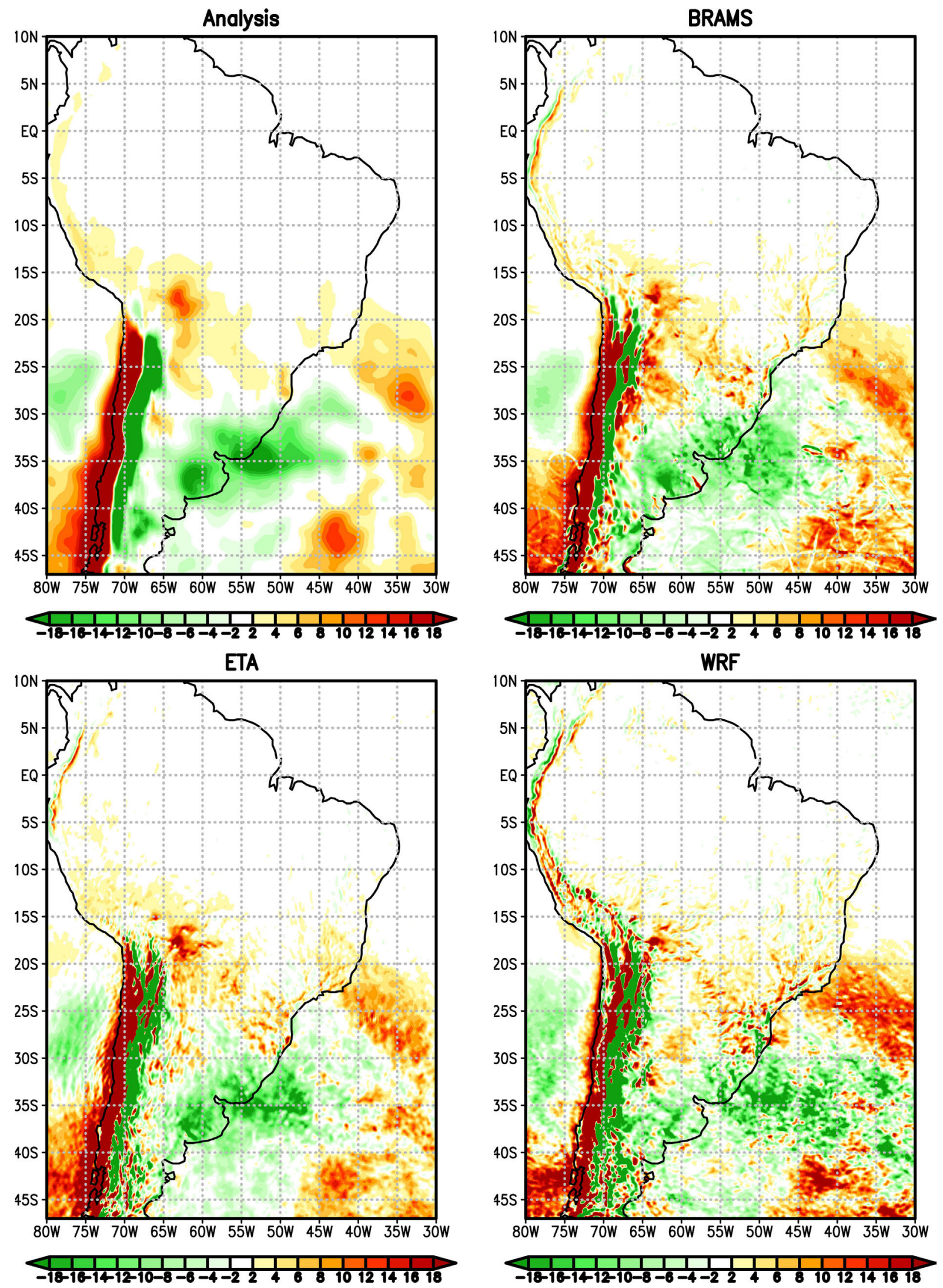

Figura 7 - Idem à Fig. 3, porém para o termo de convergência do fluxo agostrófico $\left(10^{14} \mathrm{Joule} \mathrm{s}^{-1}\right)$. 

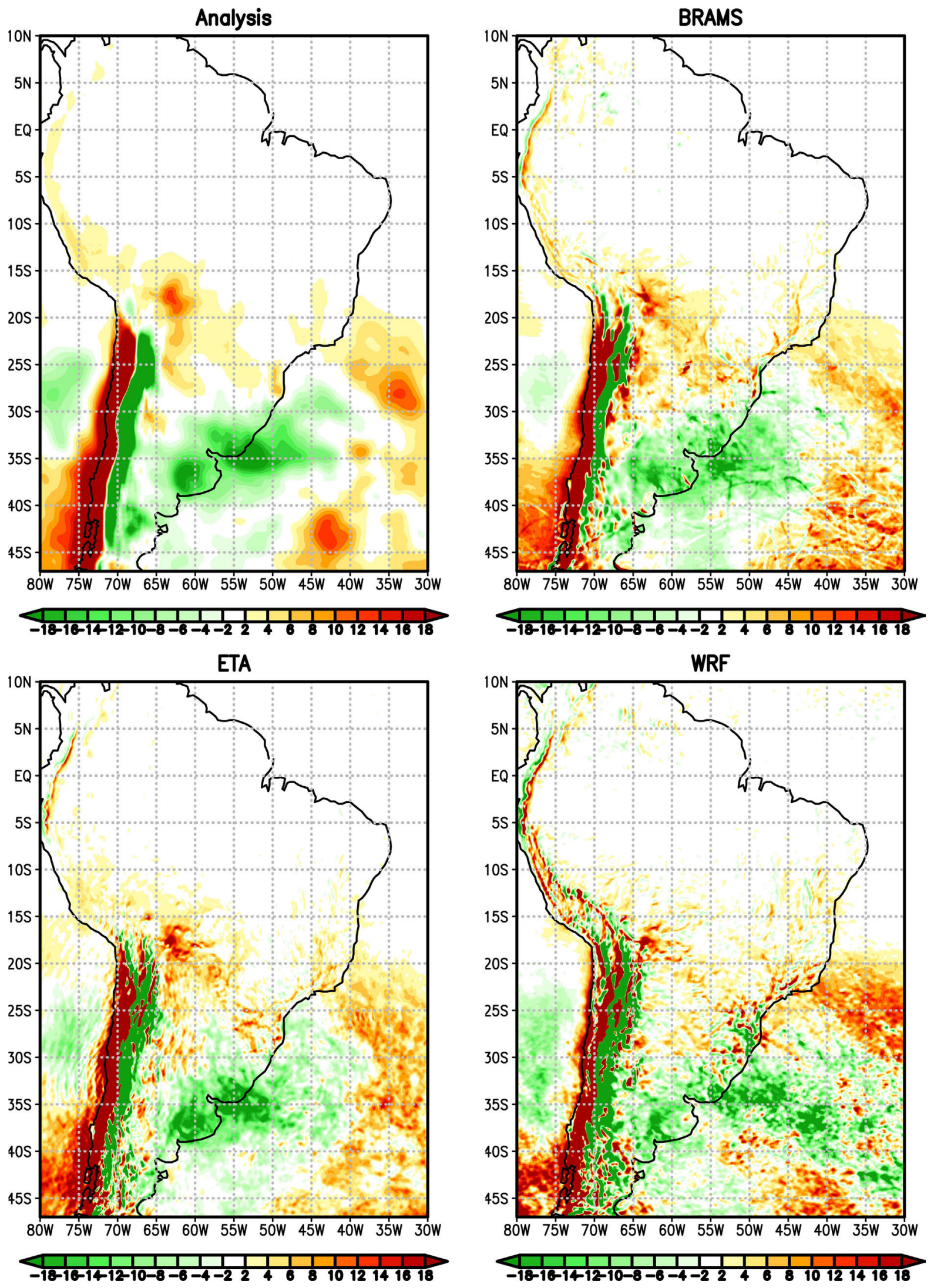

Figura 8 - Idem à Fig. 7, porém para as previsões de 48 h. 
Bias 24h
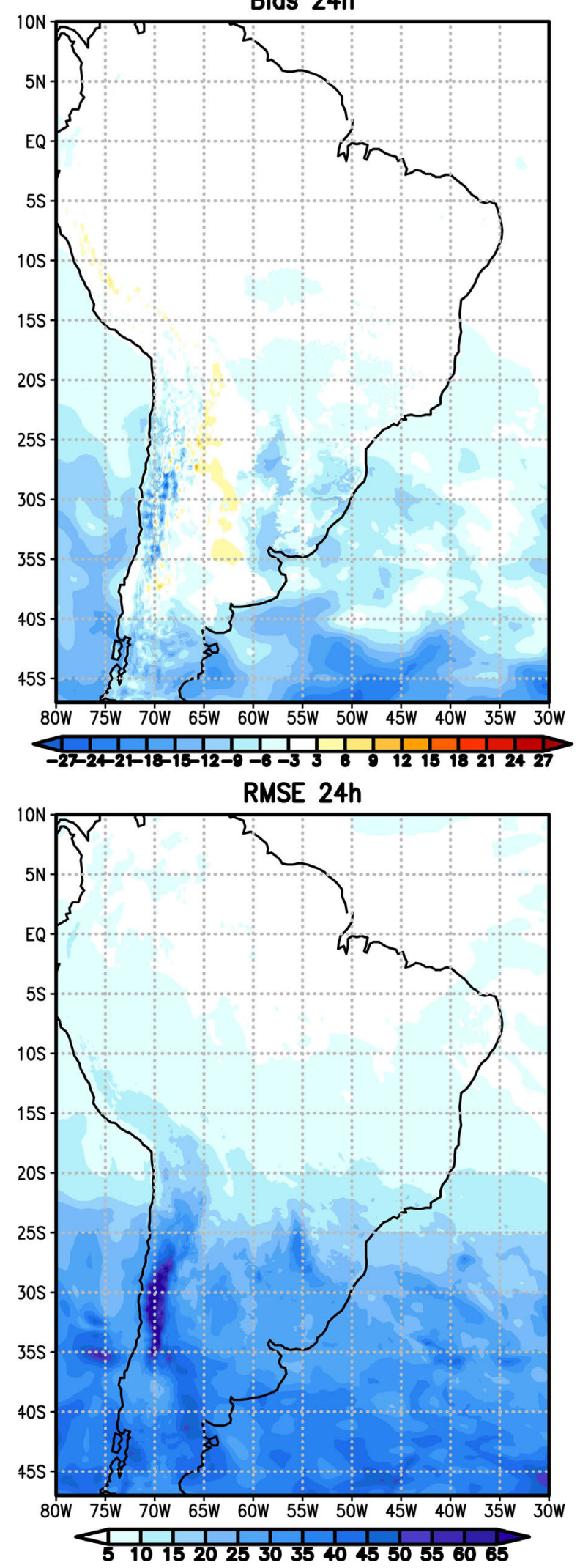

Bias 48h

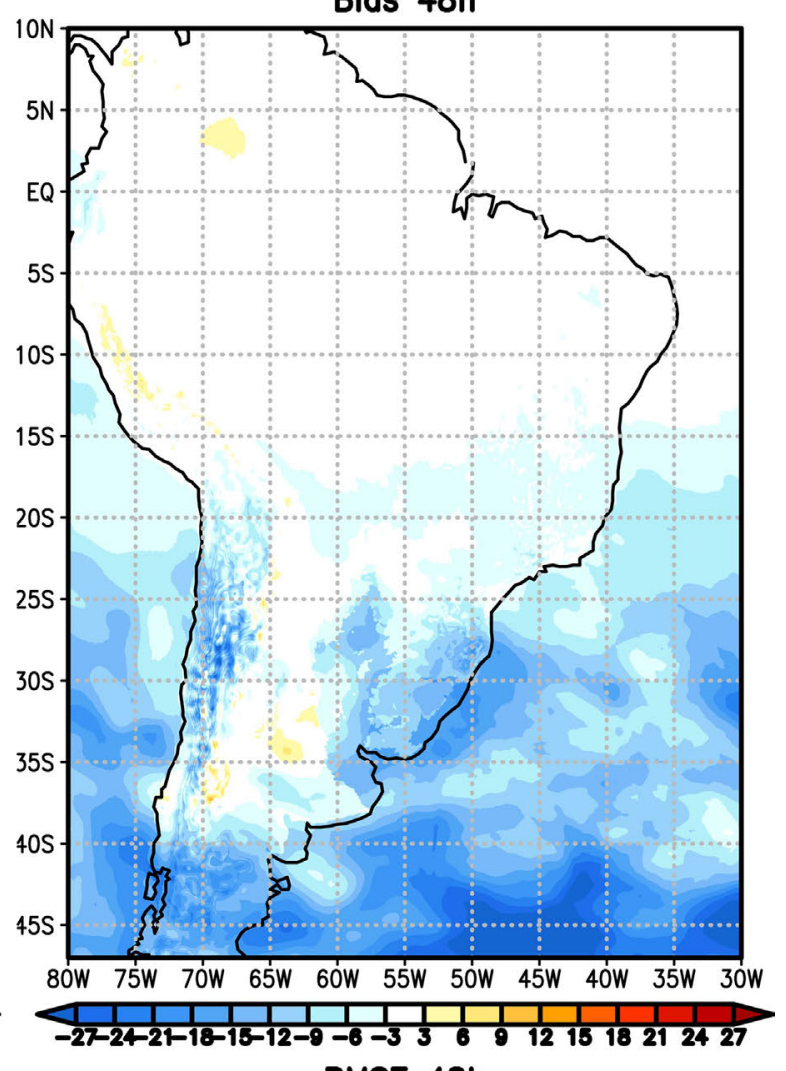

RMSE 48h

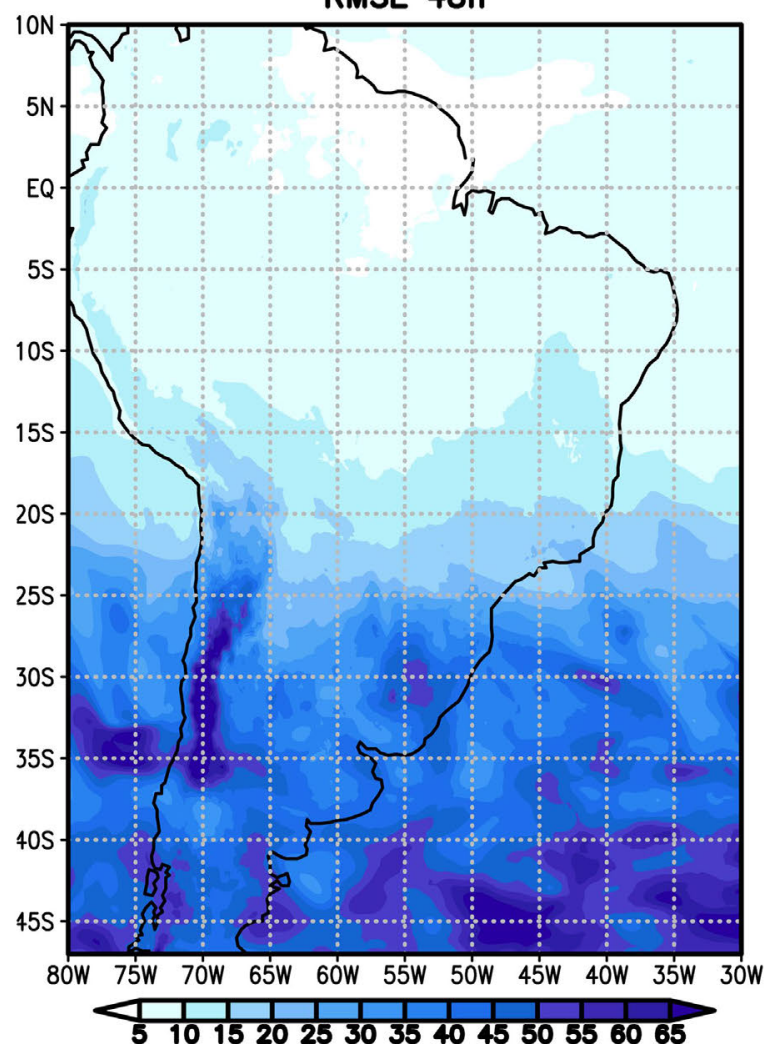

Figura 9 - Viés (painéis superiores) e RMSE (painéis inferiores) para as previsões de $24 \mathrm{~h}$ (coluna da esquerda) e $48 \mathrm{~h}$ (coluna da direita) de $K^{\text {' }}$ $\left(10^{10}\right.$ Joule $)$ do modelo BRAMS. 
Bias 24h
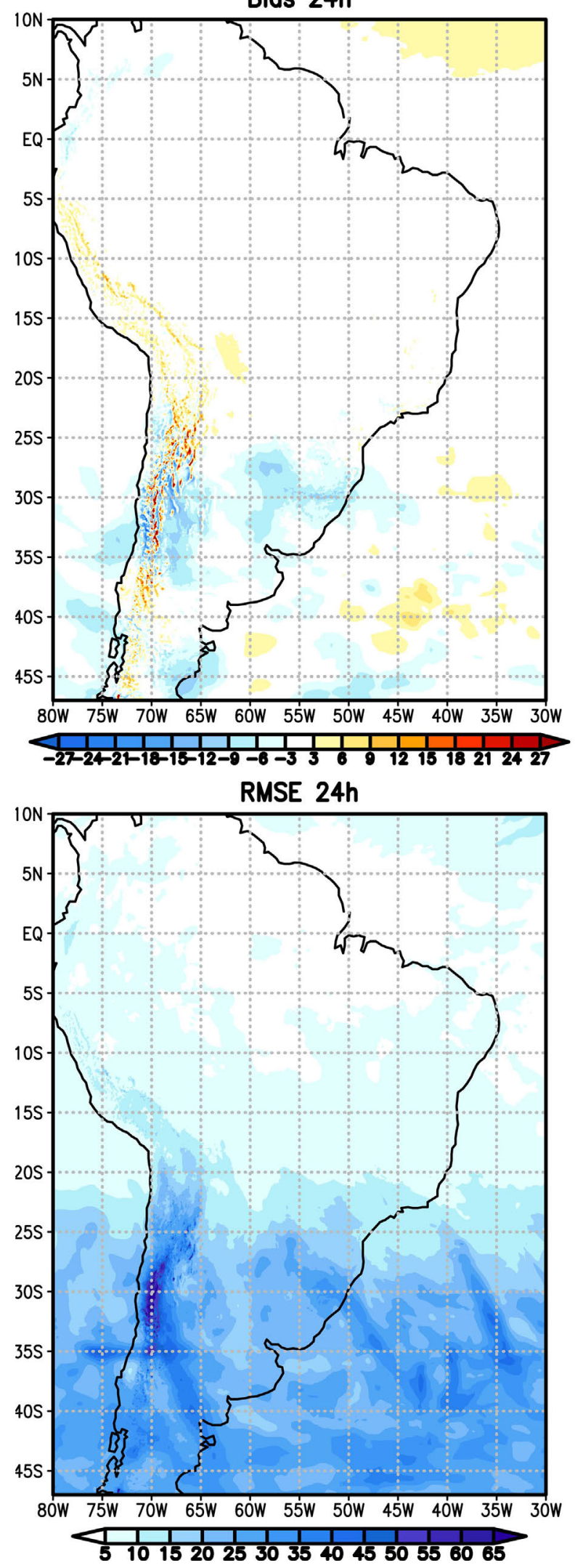

Bias 48h
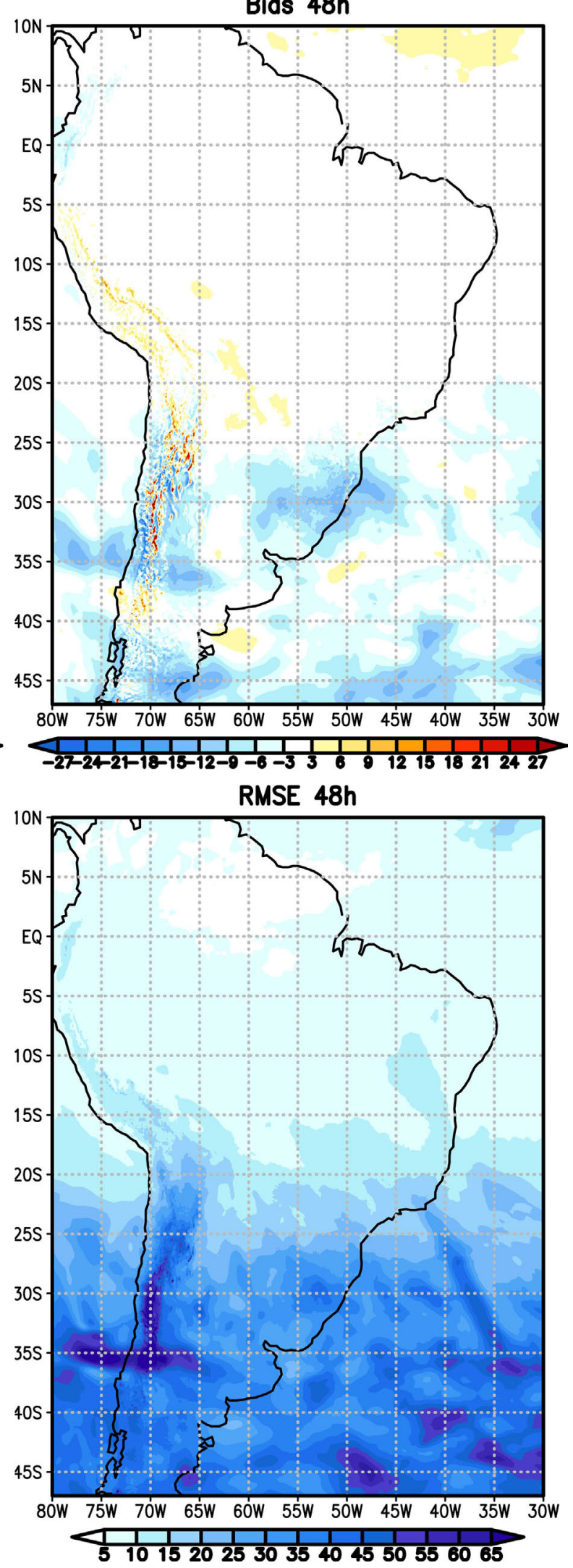

Figura 10 - Idem à Fig. 9, porém para o modelo ETA. 

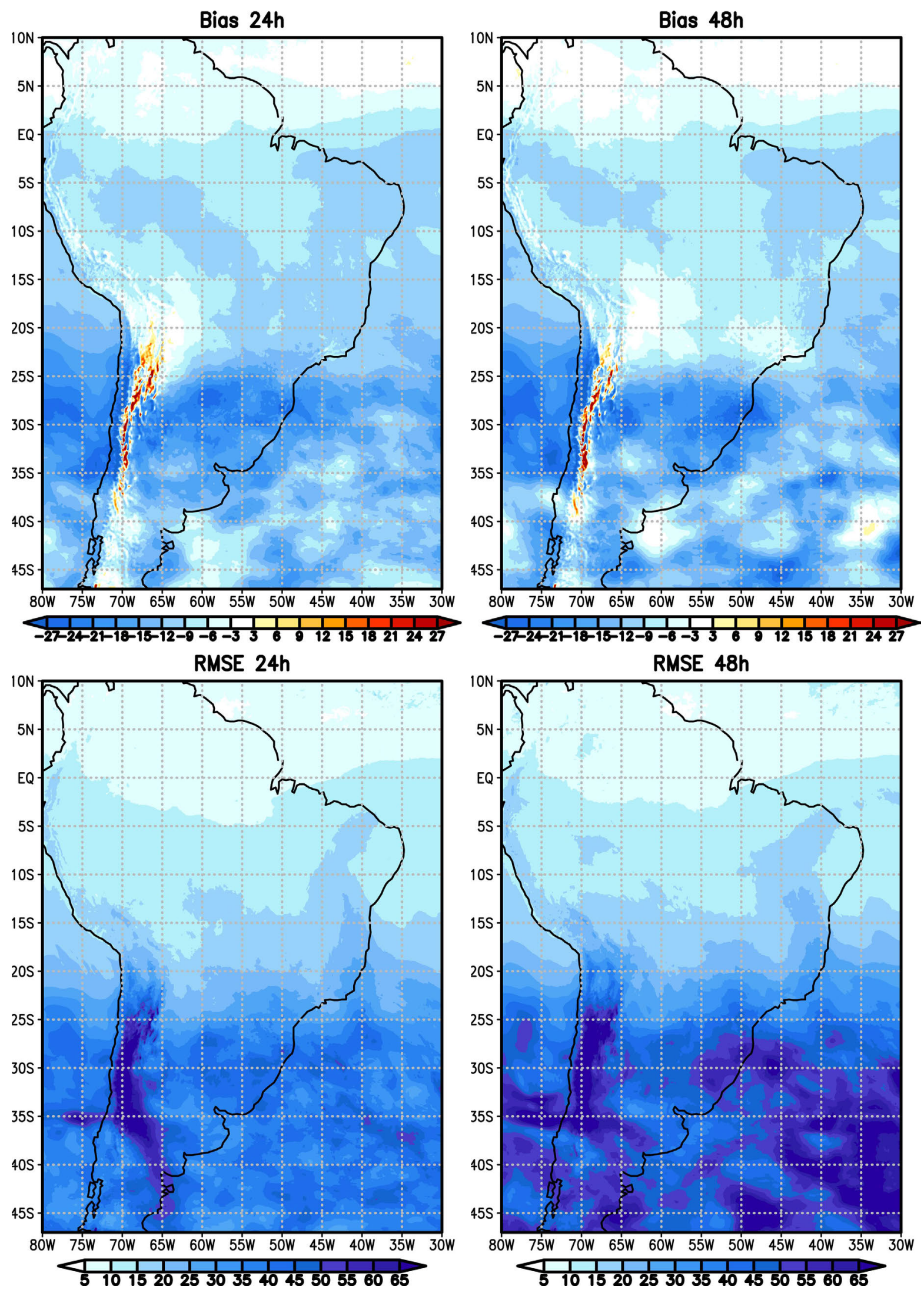

Figura 11 - Idem à Fig. 9, porém para o modelo WRF. 
Bias 24h
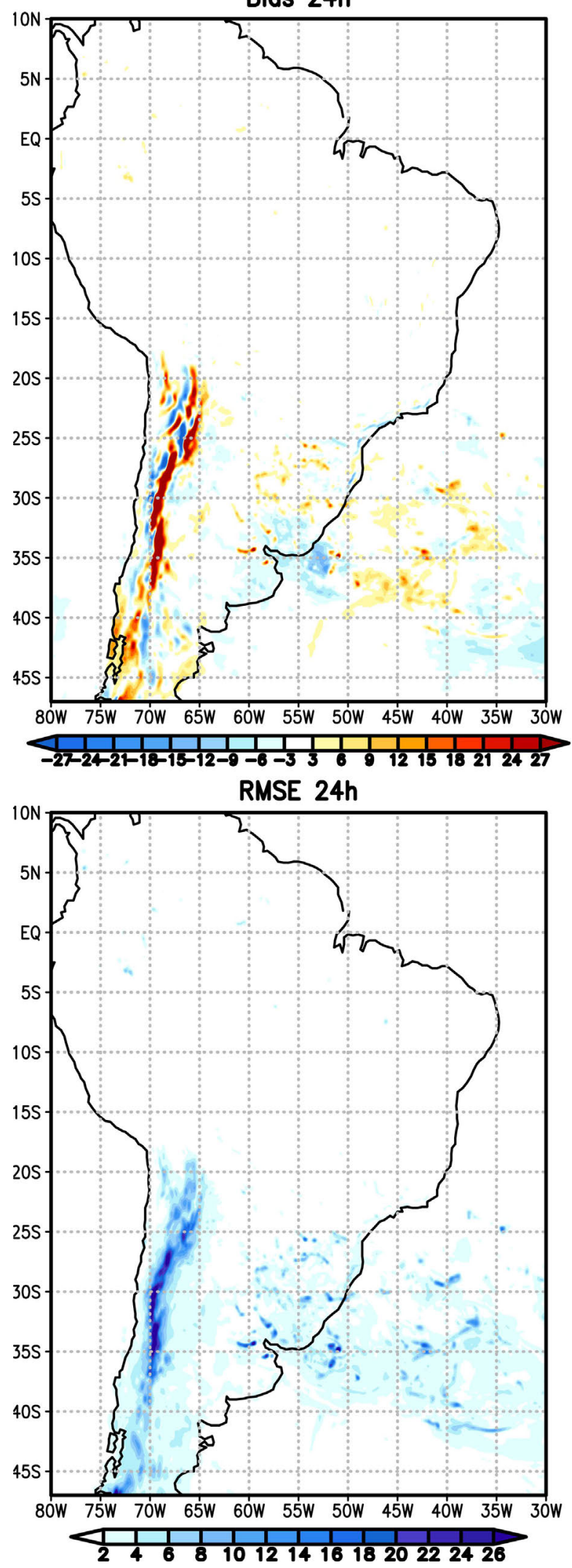

Bias 48h
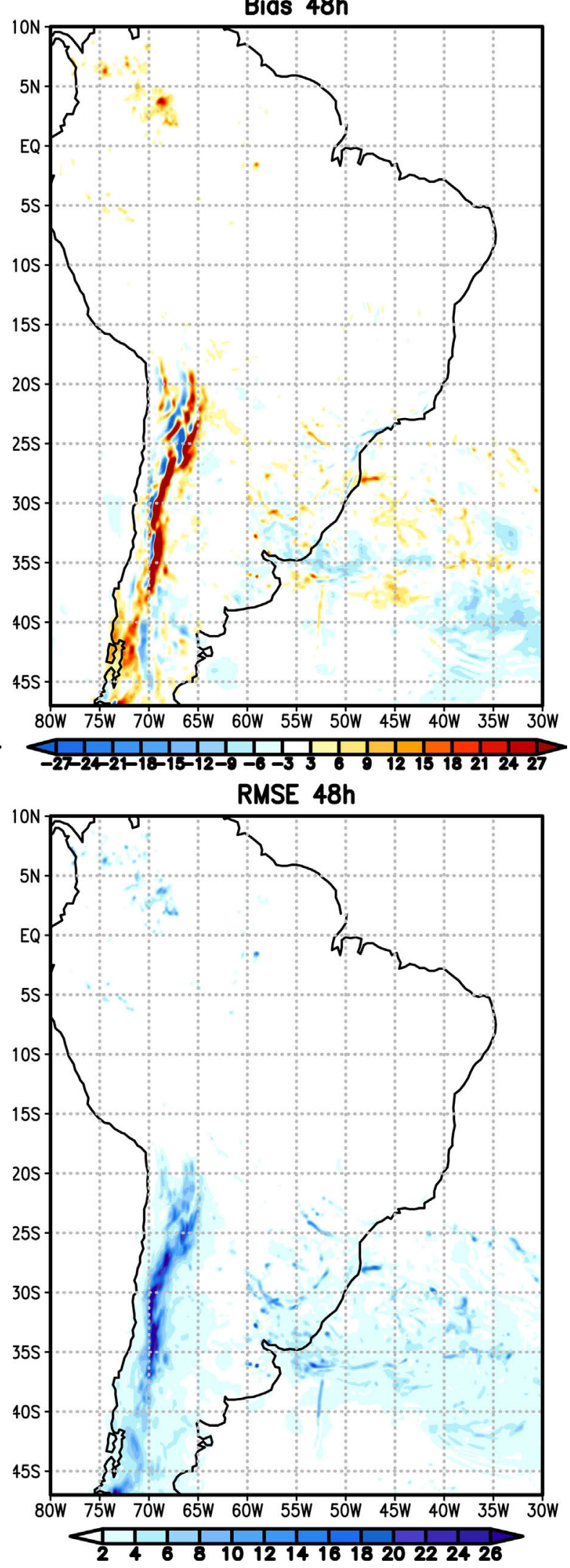

Figura 12 - Idem à Fig. 9, porém para o termo BRC. 
erros maiores de $K^{\prime}$ ao sul de $25^{\circ} \mathrm{S}$ e menores na região tropical como um todo. O WRF mostra erros maiores do que os outros dois modelos na região tropical do oceano Atlântico e parte do continente.

Para tentar entender o porquê dos modelos subestimaram $K$ ', é necessário analisar a destreza dos modelos para as previsões dos termos da equação da tendência da $K^{\prime}$, que representam os principais mecanismos atuantes na atmosfera, tais como, instabilidade baroclínica, instabilidade barotrópica e desenvolvimento corrente abaixo, representado este último mecanismo pelo termo de convergência do fluxo ageostrófico.

Analisando o viés do termo BRC do modelo BRAMS (Figs. 12a e 12b), notam-se valores significativos positivos e negativos ao sul de $20^{\circ} \mathrm{S}$, tanto nas previsões de $24 \mathrm{~h}$ como nas de $48 \mathrm{~h}$. As maiores subestimativas e superestimativas são observadas ao longo dos Andes, mostrando o problema do modelo com o efeito montanha. Sobre o oceano Atlântico e continente, podem-se ver valores positivos e negativos, porém visualmente notamse mais áreas com valores positivos. O modelo ETA (Figs. 13a e 13b) mostrou um padrão semelhante ao do BRAMS, mas as magnitudes do viés são ligeiramente menores. Por outro lado, o modelo WRF também mostrou o mesmo padrão, porém com valores maiores, e uma área mais extensa de superestimativa do termo BRC, inclusive no setor norte do domínio analisado (Figs. 14a e 14b). Esses resultados podem induzir que o modelo WRF tenderia a superestimar $K^{\prime}$ ao sul de $20^{\circ} \mathrm{S}$, mas na realidade ele subestima, como pode se ver nas Figs. 11a e 11b. Segundo Hantel e Haimberger (2000) e Haimberger e Hantel (2000), a energética tradicional contabiliza apenas a conversão baroclínica na escala da grade, desconsiderando a conversão de sub-grade. Isso sugere que, ao usar modelos de maior resolução, haverá menos conversão baroclínica de sub-grade e mais conversão na escala da grade. Isso explicaria porque, aparentemente, o viés positivo é mais frequente do que o negativo.

Como o termo BRT é menor em relação aos termos BRC e DCA, o viés e o RMSE também são pequenos em praticamente todo o domínio, exceto ao longo dos Andes ao sul de $15^{\circ} \mathrm{S}$, como pode ser visto nas Figs. 15, 16 e 17 para os três modelos. Com relação ao RMSE, os valores significativos somente aparecem ao longo dos Andes ao sul de $20^{\circ} \mathrm{S}$ nos três modelos.

Por último é analisado o termo DCA (Figs. 18, 19 e 20). O viés desse termo, para o modelo BRAMS, mostra regiões com valores positivos e negativos tanto nas previsões de 24 h (Fig. 18a) como nas de 48 h (Fig. 18b) ao sul de $20^{\circ} \mathrm{S}$. Interessante notar que as regiões onde o modelo subestima ou superestima são praticamente as mesmas nas previsões dos dois horários. Valores maiores, em módulo do viés, tanto positivos como negativos aparecem nos três modelos ao longo dos Andes ao sul de $15^{\circ}$ $\mathrm{S}$, formando um padrão de onda. Fazendo uma compar- ação visual, nota-se que os valores do viés positivos do modelo WRF são maiores e os negativos são menores, isto é, mais negativos (Figs. 20a e 20b). Comparando-se o viés desse termo com o do BRC, observa-se que para os três modelos nos dois horários, o viés do termo DCA é maior, principalmente os positivos dos modelos BRAMS e ETA, e negativo do WRF. Com relação ao WRF, como os valores do viés negativo do DCA são menores (mais negativo) do os positivos do BRC, isso pode justificar o porque esse modelo subestima $K$ ' mais fortemente do que os outros dois modelos.

O RMSE do termo DCA dos três modelos (Figs. 18, 19 e 20 - linha inferior) também mostra altos valores ao longo dos Andes ao sul de $15^{\circ} \mathrm{S}$, nos dois horários das previsões, e valores mais baixos sobre o continente e oceano ao sul de $20^{\circ} \mathrm{S}$. O modelo BRAMS (Figs. 18c e 18d) mostra, nos dois horários, um padrão muito semelhante ao do WRF (Figs. 20c e 20d), porém com valores um pouco menores. Já o modelo ETA (Figs. 19c e 19d) apresenta valores bem inferiores de RMSE comparando aos outros dois modelos, nos dois horários.

\section{Considerações Finais e Conclusões}

Uma tarefa importante para melhorar a destreza dos modelos é realizar avaliações de várias variáveis e repassar esses resultados aos profissionais de desenvolvimento dos respectivos modelos. Assim, o objetivo deste estudo foi avaliar os termos da equação da tendência da energia cinética do distúrbio $\left(K^{\prime}\right)$ nas previsões de 24 h e 48 h dos modelos regionais BRAMS, WRF e ETA, os quais eram rodados operacionalmente no Centro de Previsão de Tempo e Estudos Climáticos (CPTEC). O período de avaliação deste estudo é de 1 de junho a 31 de agosto de 2016 e o domínio a região da América do Sul. A resolução horizontal dos três modelos é de $5 \mathrm{~km}$ e a temporal é de $6 \mathrm{~h}$. A avaliação de $K^{\prime}$, assim como dos termos de conversão baroclínica e barotrópica e do termo de convergência do fluxo ageostrófico, pode representar uma nova linha de avaliação de modelos. $K$ ' está associado com a intensidade do vento (e, portanto, dos distúrbios transientes) e os termos estão associados com os principais mecanismos conhecidos de formação e intensificação dos sistemas atmosféricos que atuam nas latitudes médias.

Em geral pode-se concluir que, com relação a $K^{\prime}$ :

- Todas as previsões dos modelos subestimam nas latitudes médias, principalmente sobre os oceanos, porém o ETA é o que menos subestima e o WRF o que mais subestima;

- As previsões dos modelos têm RMSE maiores localizados nas latitudes ao sul de $20^{\circ} \mathrm{S}$ nas previsões de $24 \mathrm{~h}$ e ao sul de $15^{\circ} \mathrm{S}$ nas previsões de $48 \mathrm{~h}$.

Com relação aos termos de conversão baroclínica e barotrópica, além do termo de convergência do fluxo 
Bias 24h
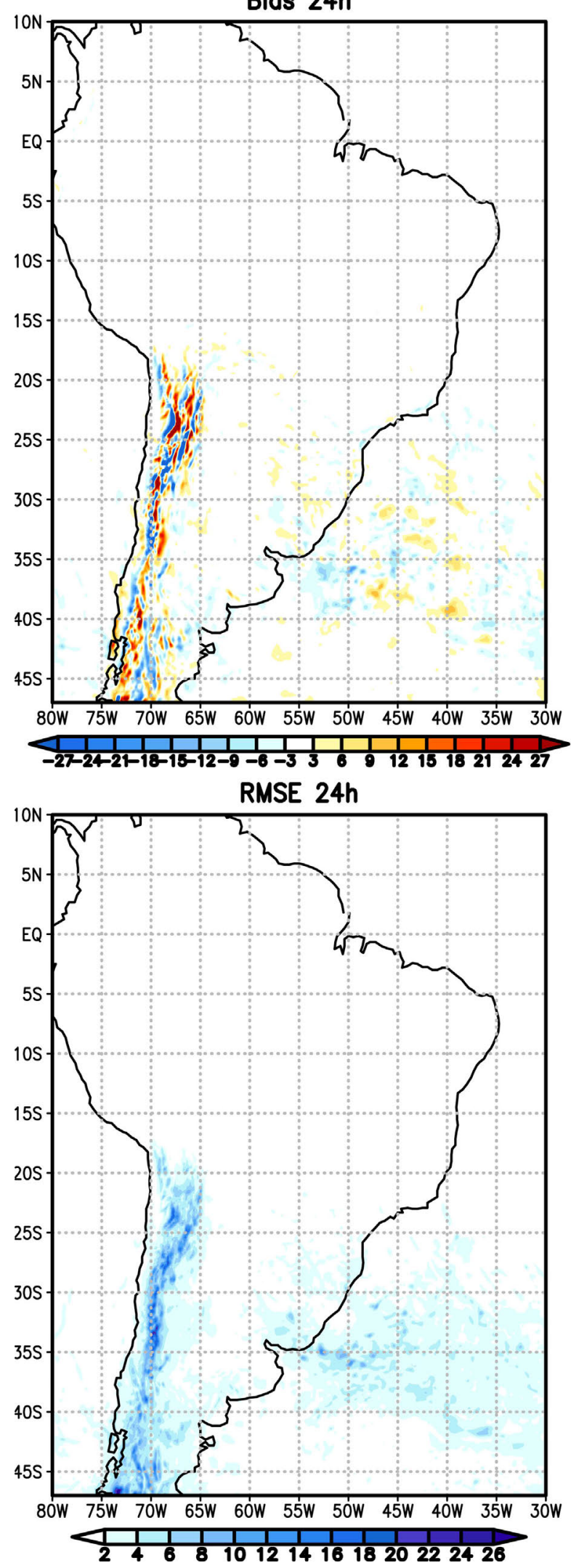

Bias 48h
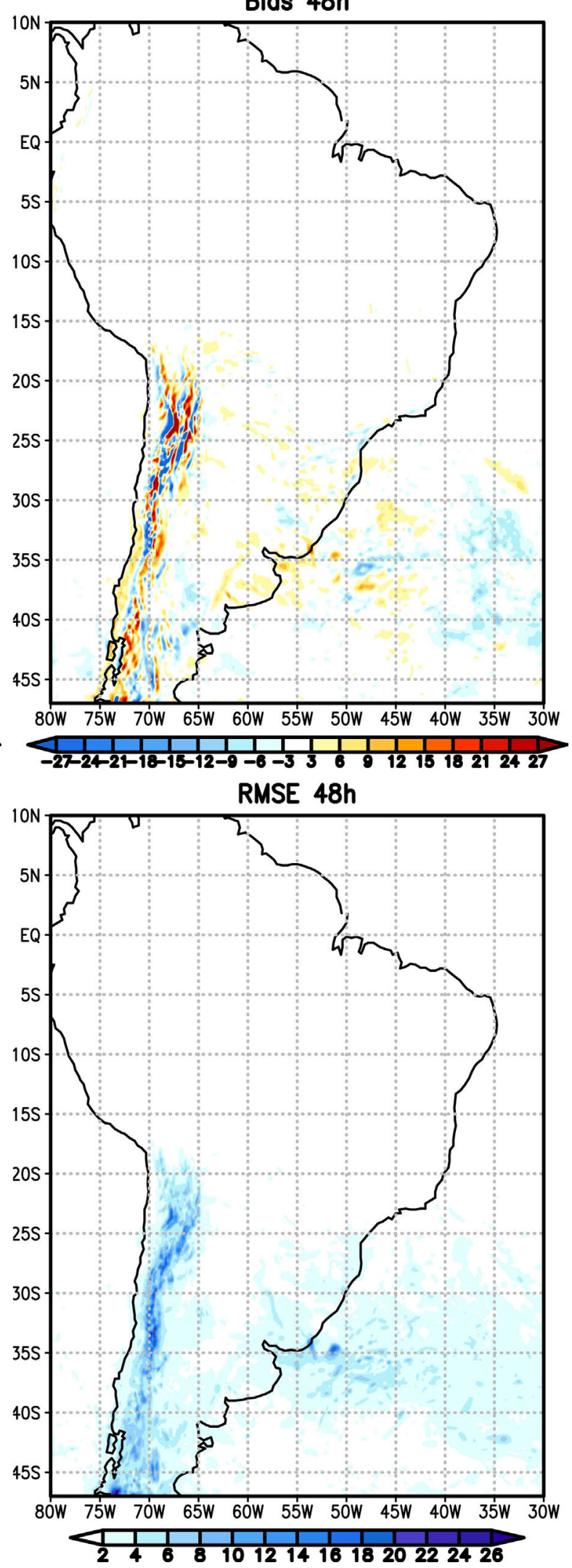

Figura 13 - Idem à Fig. 10, porém para o termo BRC. 

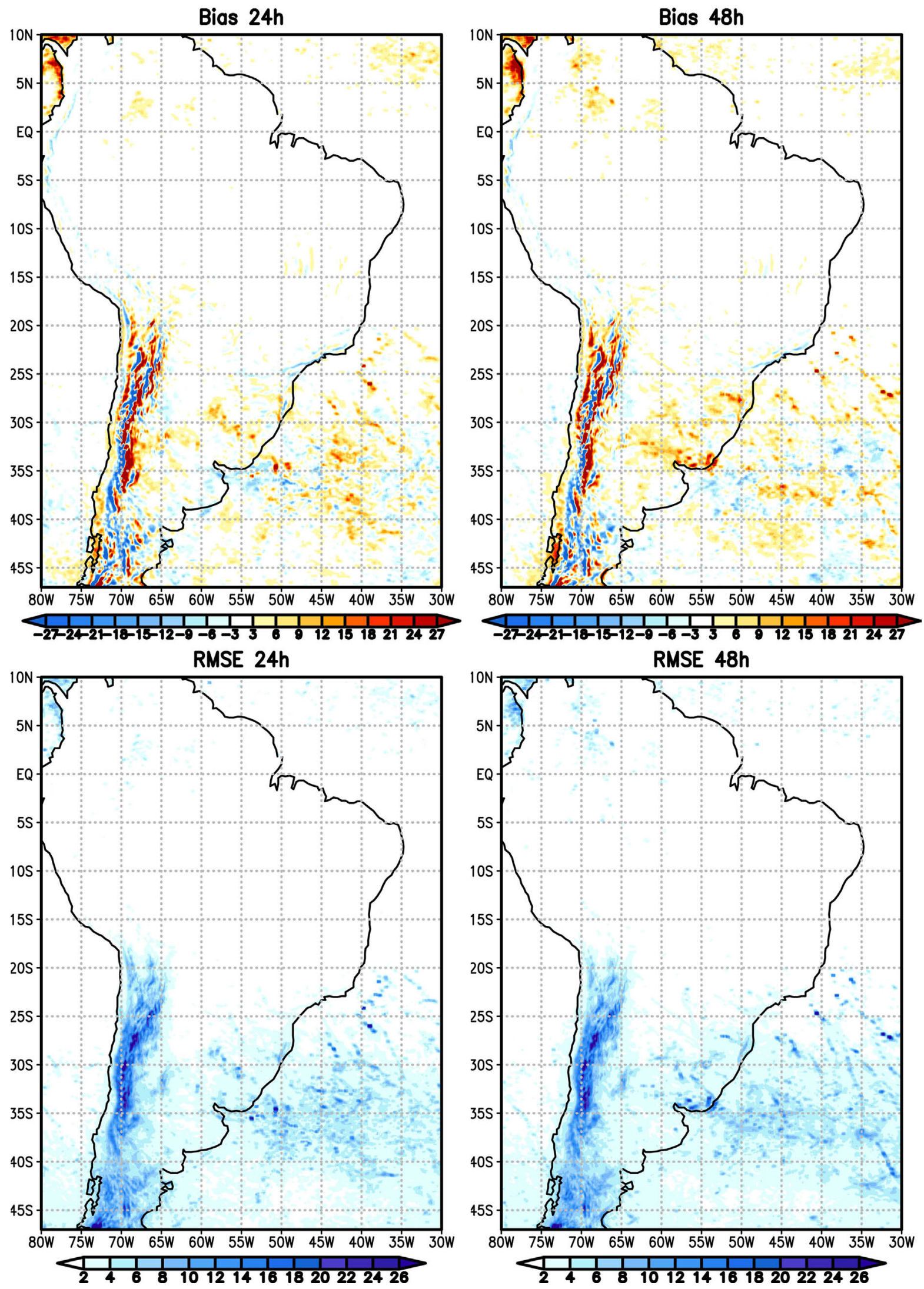

Figura 14 - Idem à Fig. 11, porém para o termo BRC. 
Bias 24h
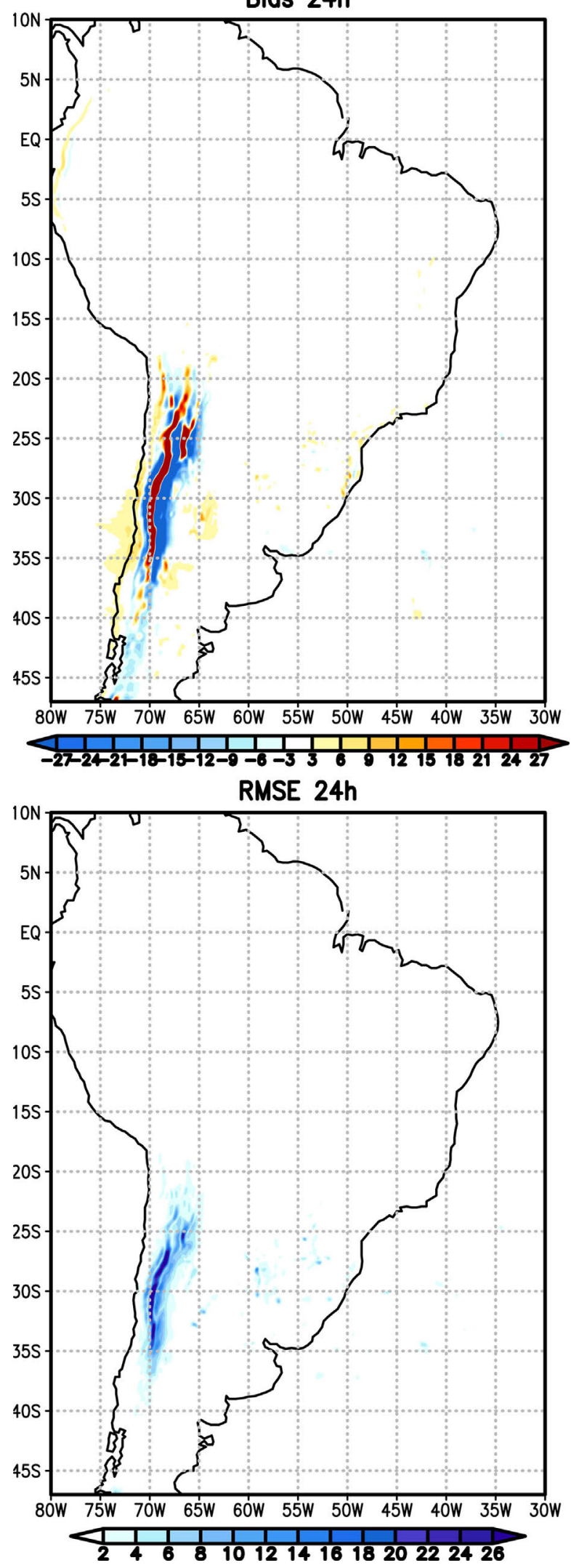

Bias 48h
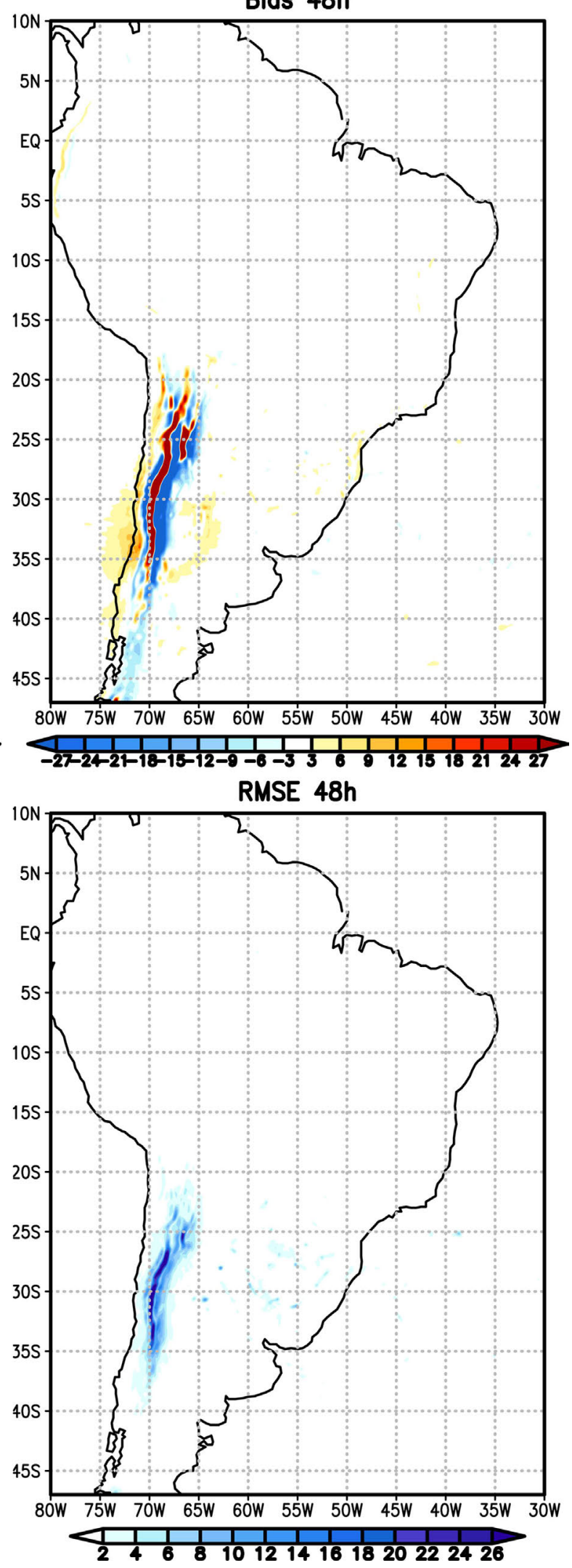

Figura 15 - Idem à Fig. 9, porém para o termo BRT. 
Bias 24h
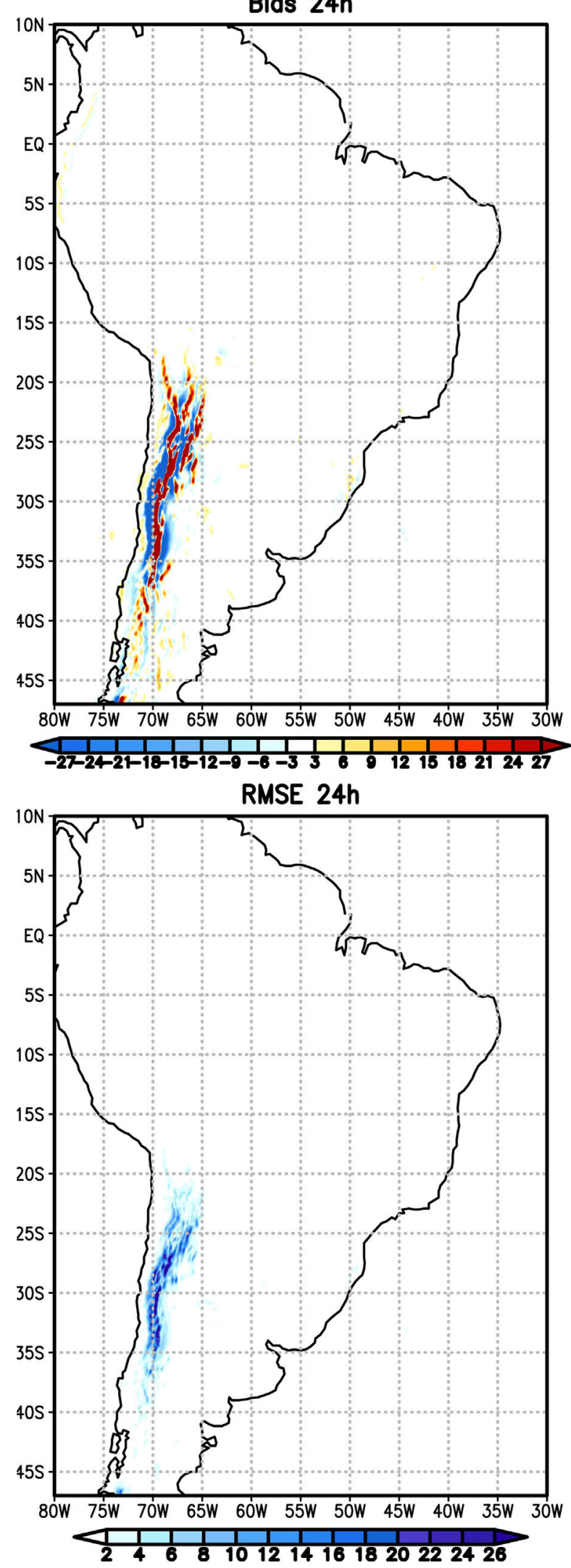

Bias 48h
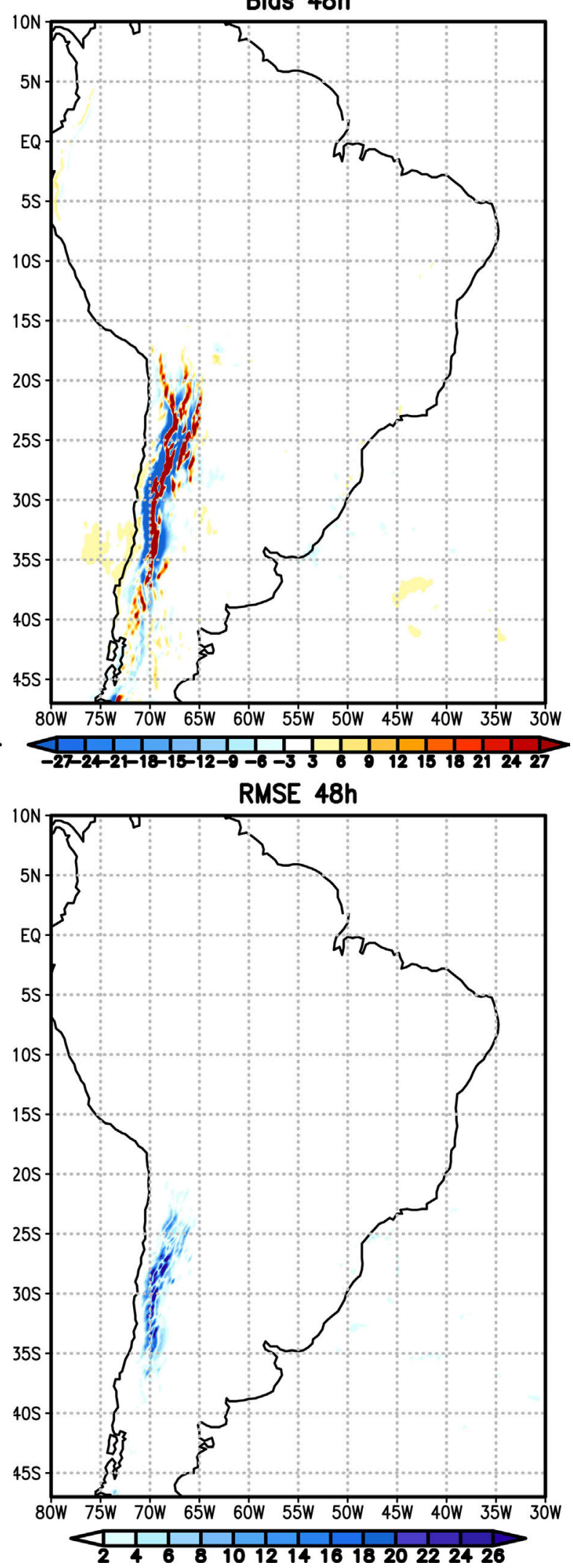

Figura 16 - Idem à Fig. 10, porém para o termo BRT. 
Bias 24h
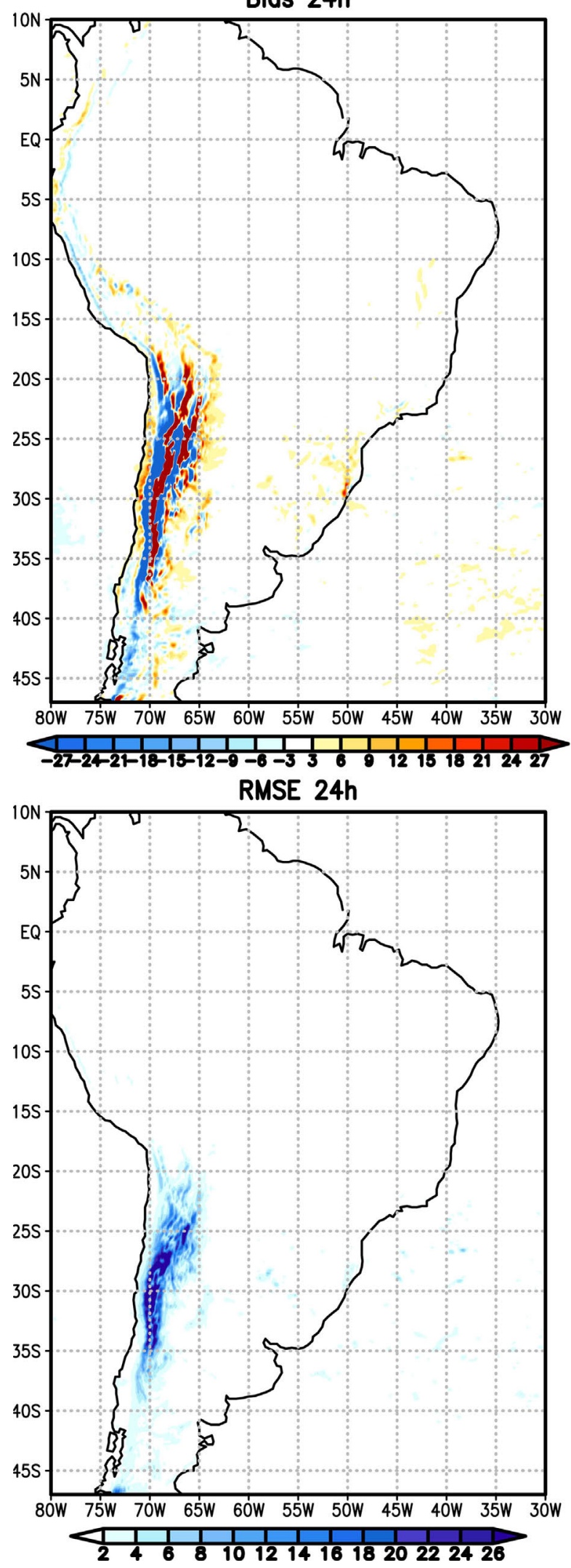

Bias 48h
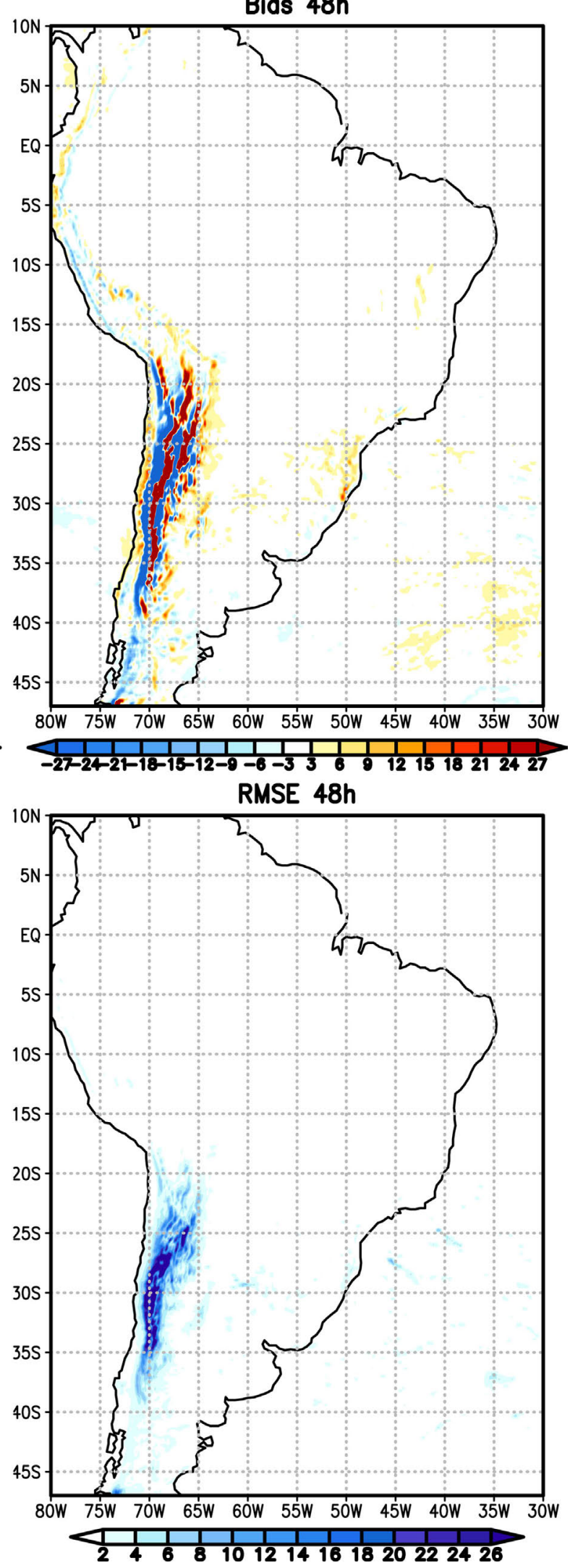

Figura 17 - Idem à Fig. 11, porém para o termo BRT. 
Bias 24h
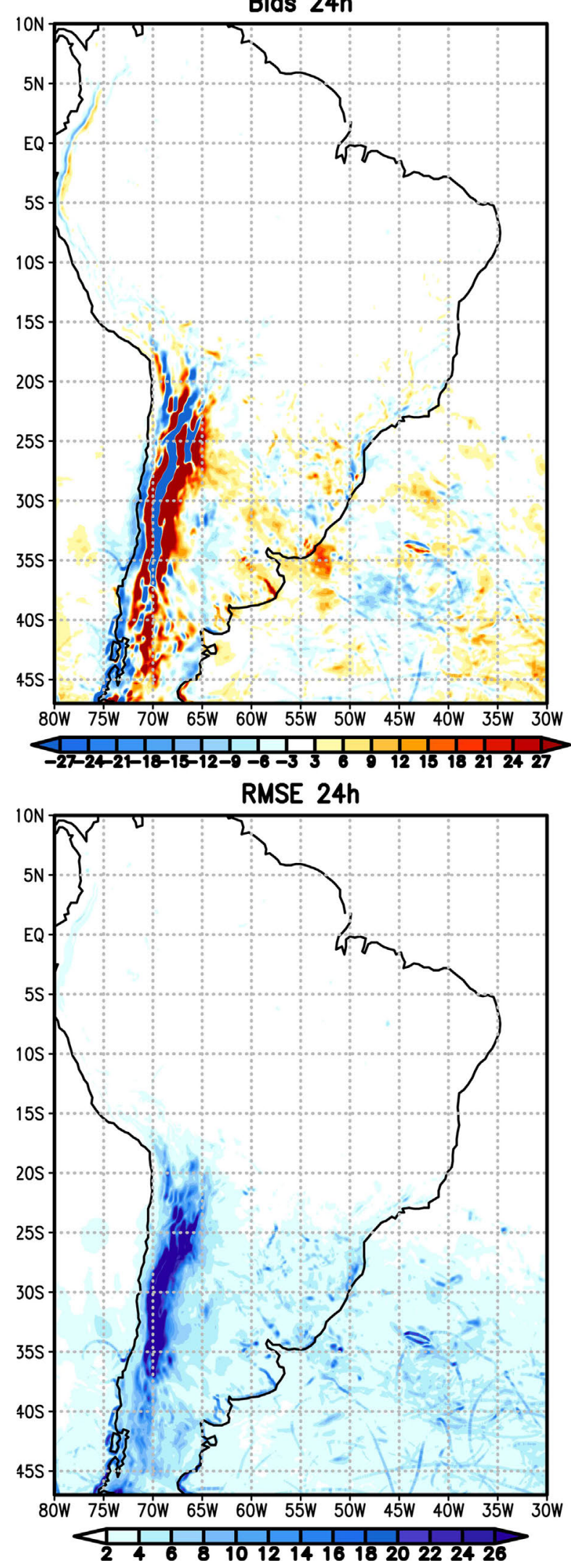

Bias 48h
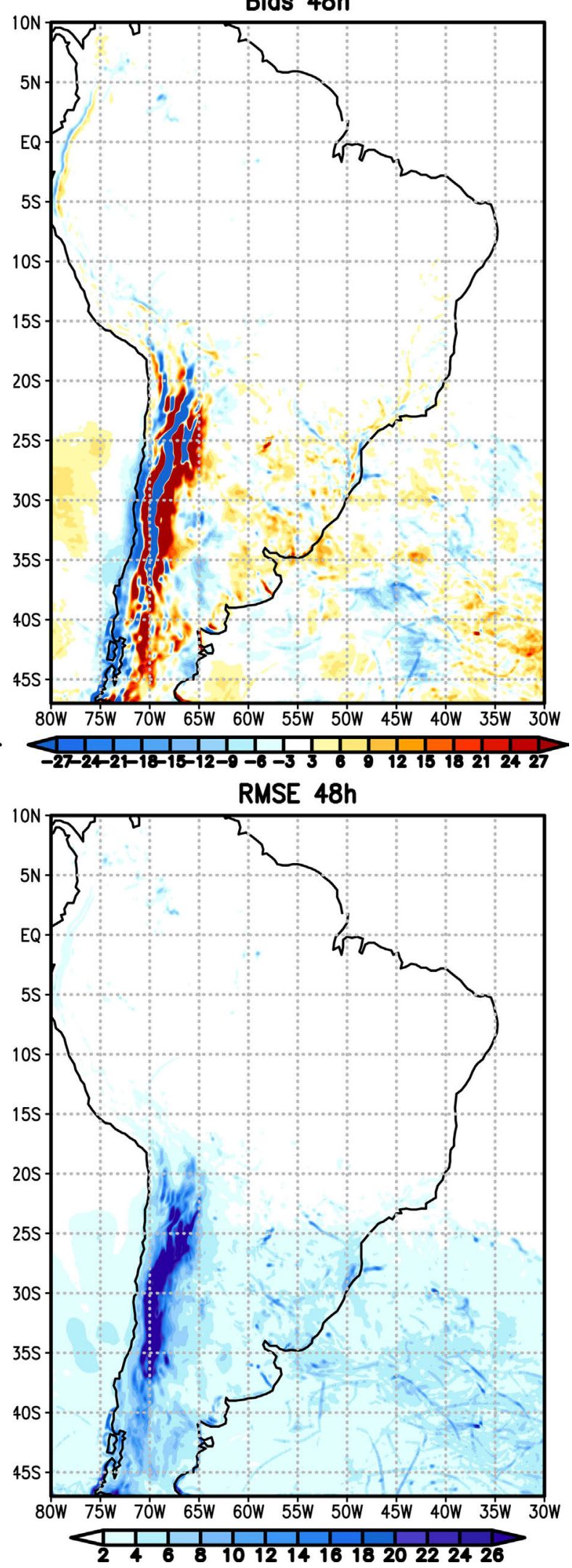

Figura 18 - Idem à Fig. 9, porém para o termo DCA. 
Bias 24h
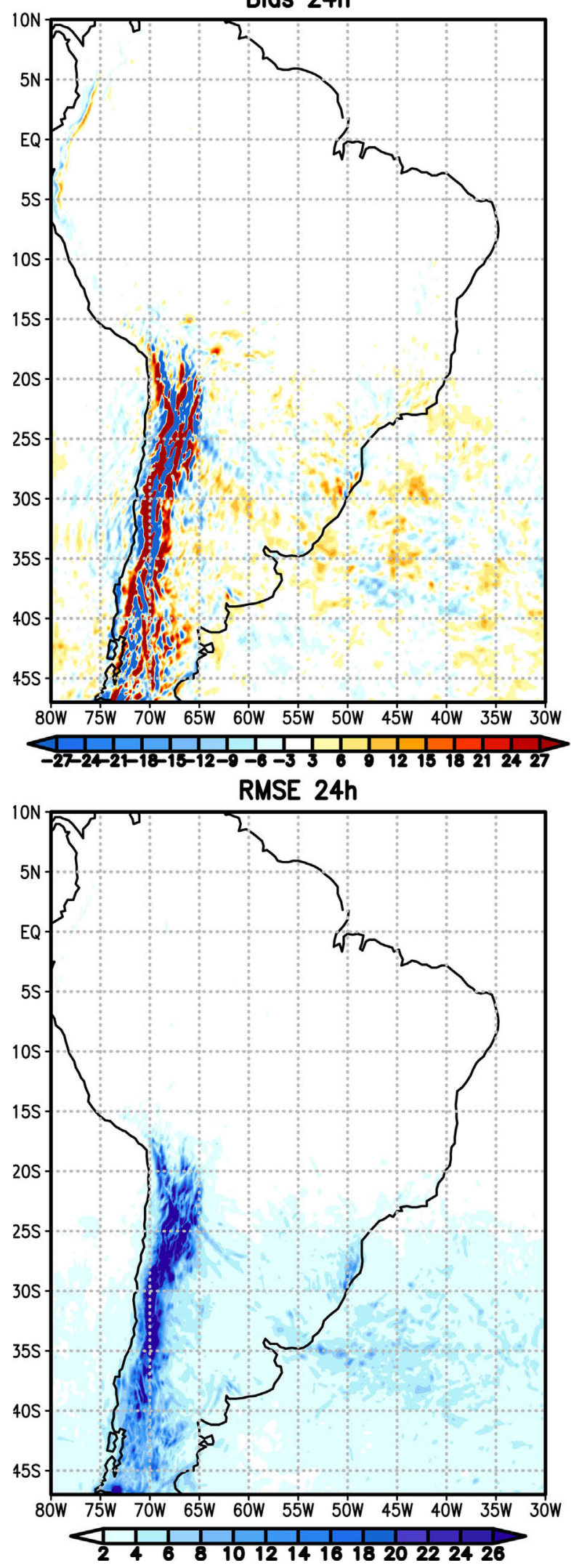

Bias 48h
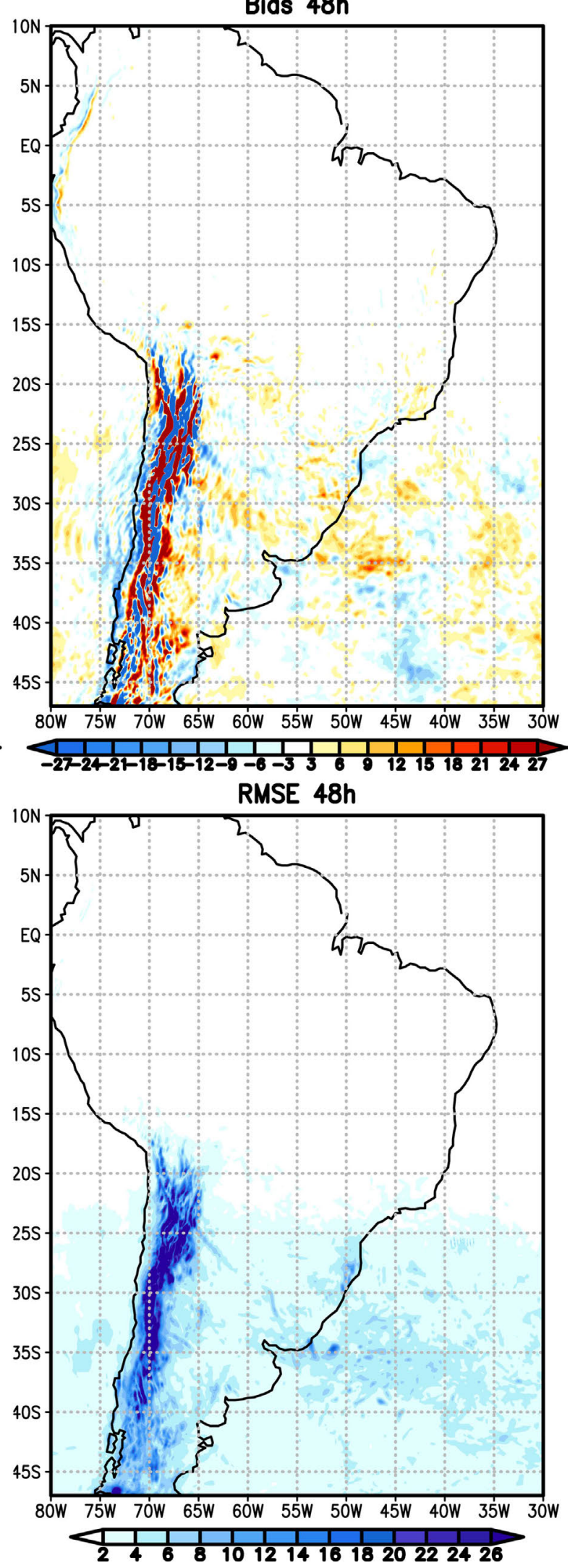

Figura 19 - Idem à Fig. 10, porém para o termo DCA. 

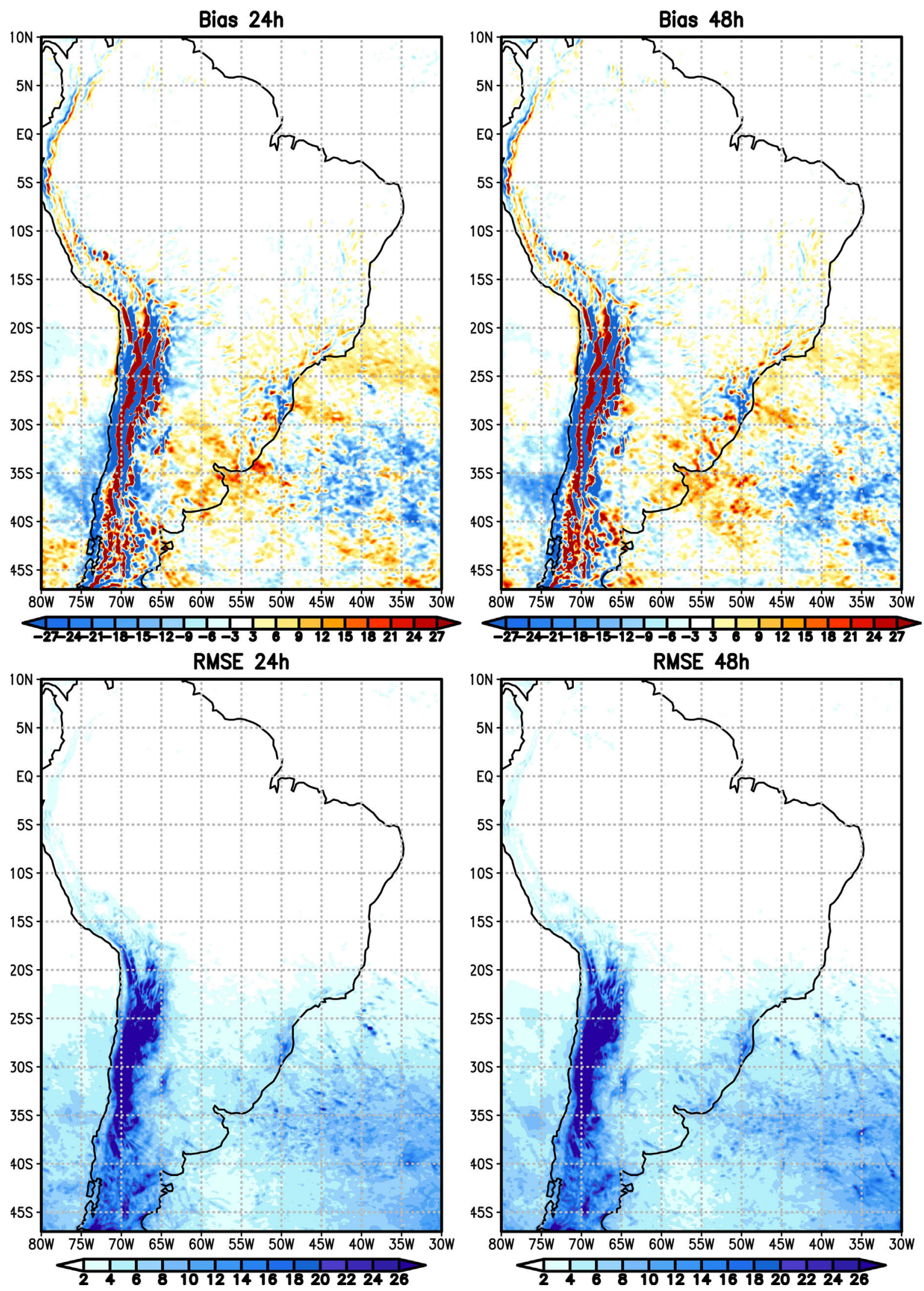

Figura 20 - Idem à Fig. 11, porém para o termo DCA. 
ageostrófico, pode-se concluir que para as previsões de $24 \mathrm{~h}$ e $48 \mathrm{~h}$ :

- As previsões dos modelos apresentam um padrão semelhante aos das análises do GFS;

- O viés e o RMSE são menores para o termo de conversão barotrópica, pois os valores desse termo são inferiores aos dos outros nas latitudes médias;

- O viés e o RMSE do termo de convergência do fluxo ageostrófico são ligeiramente superiores ao do termo de conversão baroclínica.

- $\mathrm{O}$ viés e o RMSE, associado aos três principais termos que influenciam na tendência de $K^{\prime}$, mostram-se maiores para o WRF (valores mais negativos de viés) e menores para o ETA. Portanto, esse resultado explica porque nas Figs. 1 e 2 o padrão de $K$ ' do modelo ETA (Figs. 1c e 2c) é o que mais se aproxima da análise (Figs. 1a e 2a).

Outra conclusão é que tanto as análises do GFS, quanto as previsões dos modelos têm problemas ao longo dos Andes, pois é a região onde os erros foram maiores. Esse problema pode estar relacionado com a parametrização do arrastro orográfico que pode influenciar no cálculo da velocidade do vento nos modelos, e portanto, na energia cinética e nos termos de conversão. Outro problema pode estar na representação do terreno na grade de modelo, onde as características importantes do terreno podem ser fortemente filtradas, como é o caso de uma cordilheira longa, como é os Andes, cuja atuação, como uma barreira do escoamento transversal, pode não ser bem representada pelo modelo.

Apesar dos três modelos terem subestimado $K^{\prime}$, os campos de viés e do RMSE dos termos da Equação 1 não explicam por completo essa subestimativa. Assim, supõese que outros processos, tais como liberação de calor latente e atrito, podem também não serem previstos com boa destreza, e assim, contribuírem para aumentar o erro dos modelos em prever $K^{\prime}$.

Para finalizar sugere-se que uma análise mais detalhada dos outros termos da equação da tendência da $K^{\prime}$ seja realizada para identificar qual mecanismo que o modelo não representa adequadamente, buscando assim uma previsão de vento mais próxima ao observado.

\section{Referências}

ANABOR, V.; STENSRUD, D.J.; MORAES, O.L.L. Simulation of a serial upstream-propagating mesoscale convective system event over southeastern south america using composite initial conditions. Monthly Weather Review, v. 137, n. 7, p. 2144-2163, 2009.

ANDRADE, K.M. Climatologia e Comportamento dos Sistemas Frontais sobre a América do Sul. Dissertação de Mestrado em Meteorologia, Instituto Nacional de Pesquisas Espaciais, São José dos Campos, 185 p., 2005.

BETTS, A.K.; MILLER, M.J. A new convective adjustment scheme. part ii: Single column tests using gate wave, bomex and arctic air-mass data sets. Quarterly Journal of the Royal Meteorological Society, v. 112, n. 473, p. 693709, 1986

BOER, G.J.; LAMBERT, S. The energy cycle in atmospheric models. Climate Dynamics, v. 30, n. 4, p. 371-390, 2008.

BONATTI, J.P.; RAO, V.B. Moist baroclinic instability in the development of Northe Pacific and South-American intermediate-scale disturbances. Journal of the Atmospheric Sciences, v, 44, n. 18, p. 2657-2667, 1987.

CAMPETELLA, C.; POSSIA, N. Upper-level cut-off lows in southern South America. Meteorology and Atmospheric Physics, v. 96, n. 1-2, p. 181-191, 2006.

CHANG, E.K.M. Wave packets and life cycles of troughs in the upper troposphere: Examples from the Southern Hemisphere summer season of 1984/85. Monthly Weather Review, v. 128, p. 25-50, 2000.

DOYLE, M.E.; TOMASELLA, J.; RODRÍGUEZ, D.A.; CHOU, S.C. Experiments using new initial soil moisture conditions and soil map in the Eta Model over La Plata basin. Meteorology and Atmospheric Physics, v. 121, n. 3-4, p. 119-136, 2013.

EK, M.B; MITCHELL, K.E.; LIN, Y., ROGERS, E.; GRUMMEN, P.; KOREN, V.; GAYNO, G.; TARPLEY, J.D. Implementation of NOAH land surface advances in the National Centers for Environmental Prediction operational mesoscale Eta model. Journal of Geophysical Research: Atmosfera, v. 108, n. D22, p. 8851, 2003.

FELS, S.B.; SCHWARZKOPF, M.D. The simplified exchange approximation: A new method for radiative transfer calculations. Journal of the Atmospheric Sciences, v. 32, n. 7, p. 1475-1488, 1975.

FERREIRA, S.H.S. Energética de Eventos de Jato em Baixos Níveis Durante o SALLJEX. Tese de Doutorado em Meteorologia, Instituto Nacional de Pesquisas Espaciais (INPE), São José dos Campos, 222 p., 2013. Disponível em http://urlib.net/8JMKD3MGP7W/3E2SL38.

FERRIER, B.S.; JIN, Y.; LIN, Y.; BLACK, T.; ROGERS, E.; DI MEGO, G. Implementation of a New Grid-Scale Cloud and Precipitation Scheme in the NCEP Eta Model. In: 19th Conference on Weather Analysis and Forecasting/15th Conference on Numerical Weather Prediction, San Antonio, Am Meteorol Soc., p. 280-283, 2002.

FIGUEIREDO, J.C.; SCOLAR, J. O tempo de vida médio dos sistemas convectivos de mesoescala na América do Sul. In: Congresso Brasileiro de Meteorologia, Campos de Jordão, p. 984-986, 1996.

FUENZALIDA, H.; SÁNCHEZ, R.; GARREAUD, R. A climatology of cutoff lows in the Southern Hemishere. Journal of Geophysical Research: Atmosfera, v. 110, n. D. 18, p. D18101, 2005.

FUNATSU, B.M.; GAN, M.A.; CAETANO NETO, E.S. A case of orographic cyclogenisys over South America. Atmosfera, v. 17, n. 2, p. 91-113, 2004.

GAN, M.A.; PIVA, E.D. Energetics of a southearstern Pacific cut-off low. Atmospheric Science Letters, v. 14, n. 4, p. 272-280, 2013.

GAN, M.A.; RAO, V.B. Energetics of the high frequency disturbances over south America. Brazilian Journal of Geophysics, v. 17 , n. 1 p. 21-27,1999. 
GAN, M.A.; RAO, V.B. Surface cyclogenesis over South America. Monthly Weather Review, v. 119, n. 5, p. 1293-1303, 1991.

GAN, M.A.; PIVA, E.D. Energetics of southeastern Pacific cutoff lows. Climate Dynamics, v. 46, n. 11-12, p. 3453-3462, 2016.

GEVAERD, R.; FREITAS, S.R. Operational estimation of soil moisture for initialization of numerical prediction models of the atmosphere. Part I: Description of methodology and validation, 2006. Revista Brasileira de Meteorologia, v. 21, n. 3, p. 1-15, 2006.

GRELL, G.A.; DÉVÉNYI, D. A generalized approach to parameterizing convection combining ensemble and data assimilation techniques. Geophysical Research Letters, v. 29, n. 14, p. 38-1-38-4, 2002.

GRELL, G.A.; FREITAS, S.R A scale and aerosol aware stochastic convective parameterization for weather and air quality modeling. Atmospheric Chemistry Physics, v. 14, n. 10, p. 5233-5250, 2014.

HAIMBERGER, L.; HANTEL, M. Implementing convection into Lorenz's global cycle: Parte II. A new estimate of the conversion rate into kinetic energy. Tellus, v. 52A, n. 1, p. 75-92, 2000.

HALLAK, R. Aspectos Dinâmicos e Simulação Numérica da Formação e Evolução de um Vórtice de Ar Frio. Dissertação de Mestrado em Meteorologia, Instituto de Astronomia, Geofísica e Ciências Atmosféricas, 2000.

HANTEL, M.; HAIMBERGER, L. Implementing convection into Lorenz's global cycle: parte I. gridscale averaging of the energy equations. Tellus, v. 52A, n. 1, p. 66-74, 2000.

IACONO, M.J.; DEÇAMERE, J.S.; MLAWER, E.J.; SHEPHARD, M.V.; CLOGH, S.A., COLLINS, W.D. Radiative forcing by longlived greenhouse gases: Calculations with the AER radiative transfer models. Journal of Geophysical Research, v. 113, n. D13, p. D13103, 2008.

IWABE, C.M.N.; da ROCHA, R.P. An event of stratospheric air intrusion and its associated secondary surface cyclogenesis over the South Atlantic Ocean. Journal of Geophysical Research, v. 114, n. D9, p. D09101, 2009.

JANJIC, Z.I. The step-mountain coordinate: physical package. Monthly Weather Review, v. 118, n. 7, p. 1429-1443, 1990.

JANJIC, Z.I. The step-mountain eta coordinate model: Further developments of the convection, viscous sublayer, and turbulence closure schemes. Monthly Weather Review, v. 122, n. 5, p. 927-945, 1994.

KOUSKY, V.E. Frontal influences on Northeast Brasil. Monthly Weather Review, v. 107, n. 9, p. 1140-1153, 1979.

KOUSKY, V.E.; GAN, M.A. Upper tropospheric cyclonic vortices in the subtropical South Atlantic. Tellus, n. 6, v. 33, p. 538-551, 1981.

LACIS, A.A.; HANSEN, J.E. A parameterization of the absorption of solar radiation in the earth's atmosphere. Journal of the Atmospheric Sciences, v. 31, n. 1, p. 118-133, 1974.

LIU, Y.; WU, G.; REN, R. Relationship between the subtropical anticyclone and diabatic heating. Journal of Climate, v. 17, n. 4, p. 682-698, 2004.
LORENZ, E.N. The Nature and Theory of the General Circulation of the Atmosphere. World Meteorological Organization, Geneva, 161 p., 1967.

MELLOR, G.L.; YAMADA, T. Development of a turbulence closure model for geophysical fluid problems. Reviews of Geophysical Physics and Space Physics, v. 20, n. 4, p. 851-875, 1982.

MESINGER, F. A blocking technique for representation of mountains in atmospheric mo els. Rivista di Meteorologia Aeronautica, v. 44, n. 1-4, p. 195-202, 1984.

MESINGER, F.; CHOU, S.C.; GOMES. J.L.; JOVIC, D,; Bastos, P., et al. An upgraded version of the Eta model. Meteorology and Atmospheric Physics, v. 116, n. 3, p. 63-79, 2012.

MLAWER, E.J.; TAUBMAN, S.J.; BROWN, P.D.; IACONO, M.J.; CLOUGH, S.A. Radiative transfer for inhomogeneous atmospheres: RRTM, a validated correlated-k model for the longwave. Journal of Geophysical Research, v. 102, n. D 14, p. 16663-16682, 1997.

MORAIS M.D.C. Vórtices Ciclônicos de Altos Níveis que Atuam no Nordeste do Brasil: Estudo Observacional e Numérico. Tese de Doutorado, Instituto Nacional de Pesquisas Espaciais, São José dos Campos, 2016.

MOREIRA, D.S.; FREITAS, S.R.; BONATTI, J.P.; MERCADO, L.M.; ROSÁRIO, N.M.E.; LONGO, K.M.; MILLER, J.B.; GLOOR, M.; GATTI, L.V. Coupling between the Jules land-surface scheme and the CCATT-BRAMS atmospheric chemistry model (JULES-CCATT-BRAMS1.0): applications to numerical weather forecasting and the co 2 budget in south america. Geoscientific Model Development, v. 6, n. 4, p. 1243-1259, 2013.

MÜLLER, G.V.; GAN, M.A.; PIVA, E.D. Energetics of wave propagation leading to frost events in South America: Extratropical latitudes. Atmospheric Science Letters, v. 18, n. 8, p. 342-348, 2017.

NIELSSEN-GAMMON, J.W. Dynamical conceptual models of upper-level mobile trough formation: Comparison and application. Tellus, v. 47A, n. 5, p. 705-721, 1995.

OLIVEIRA, A.S. Interações entre Sistemas Frontais na América do Sul e a Convecção na Amazônia. Dissertação de Mestrado, Instituto Nacional de Pesquisas Espaciais, São José dos Campos, 134 p, 1986. Disponivel em http:// urlib.net/sid.inpe.br/MTC-m13@80/2005/08.22.13.12.

OLSON, J.S. Global Ecosystem Framework-Definitions. Internal Rep., USGS EROS Data Center, 37 pp, 1994.

ORLANSKI, I., SHELDON, J.P. A case of downstream baroclinic development over Western North America. Monthly Weather Review, v. 121, n. 11, p. 2929-2950. Nov 1993.

ORLANSKI, I.; KATZFEY, J. The life cycle of a cyclone wave in the Southern Hemisphere. 1. Eddy energy budget. Journal of the Atmospheric Science, v. 48, p. 1972-1998, 1991.

PINHEIRO, H.R. Validação do Método TRACK para Identificação Objetiva dos Vórtices Ciclônicos de Altos Níveis em Regiões Subtropicais. Dissertação de Mestrado em Meteorologia, Instituto Nacional de Pesquisas Espaciais, São José dos Campos, 132 p., 2010. 
PIVA, E.D.; GAN, M.A.; RAO, V.B. Energetics of winter troughs entering South America. Monthly Weather Review, v. 138, n. 4, p. 1084-1103, 2010.

ROSA, M.B.; FERREIRA, N.J.; GAN, M.A.; MACHADO, L.H.R. Energetics of cyclogenesis events over the southern coast of Brazil. Revista Brasileira de Meteorologia, v. 28, n. 3, p. 231-245, 2013.

REBOITA, M.S. Ciclones Extratropicais Sobre o Atlântico Sul: Simulação Climática e Experimentos de Sensibilidade. Tese de Doutorado em Meteorologia, Instituto de Astronomia, Geofísica e Ciências Atmosféricas, 359 p., 2008.

REBOITA, M.S.; DA ROCHA, R.P.; AMBRIZZI, T.; SUGAHARA S. South Atlantic Ocean cyclogenesis climatology simulated by regional climate model (RegCM3). Climate Dynamics, v. 35, n. 7, p. 1331-1347, 2009.

REYNOLDS, R.W.; RAYNER, N.A.; SMITH, T.M.; STOKES, D.C.; WANG W. An improved in situ and satellite SST analysis for climate. Journal of Climate, v. 15, n. 13, p. 1609-1625, 2002.

RODRIGUES, M.L.G.; FRANCO, D.; SUGAHARA, S. Climatologia de frentes frias no litoral de Santa Catarina. Revista Brasileira de Geofísica, v. 22, n. 2, p. 135-151, 2004.
SKAMAROCK, W.C. Evaluating mesoscale NWP models using kinetic energy spectra. Montlhy Weather Review, v. 132, n. 12, p. 3019-3032, 2004.

SEAGER, R.; MUTUGUDDE, R., NAIK, N.; CLEMENT, A.; GORDON, N.; MILLER, J. Air-sea interaction and the seasonal cycle of the subtropical anticyclones. Journal of Climate, v.16, n. 12, p. 1948-1966, 2003.

SESTINI, M.; REIMER, E.; VALERIANO, D.; ALVALÁ, R.S.; MELLO, E.; CHAN, C.; NOBRE, C. Mapa de cobertura da terra da Amazônia Legal para uso em modelo meteorológicos. In: XI Simpósio Brasileiro de Sensoriamento Remoto, Belo Horizonte, p. 2901-2906, 2003.

TREMBACK, C.J.; POWELL, J.; COTTON, W.R.; PIELKE, R.A. The forward in-time upstream advection scheme: Extension to higher orders. Montlhy Weather Review, v. 115 , n. 2 , p. $540-555,1987$.

ZOBLER, L. Global Soil Types, 1-Degree Grid (Zobler). ORNL DAAC, Oak Ridge, 1999. Available online at http:// www.daac.ornl.gov.

License information: This is an open-access article distributed under the terms of the Creative Commons Attribution License (type CC-BY), which permits unrestricted use, distribution and reproduction in any medium, provided the original article is properly cited. 